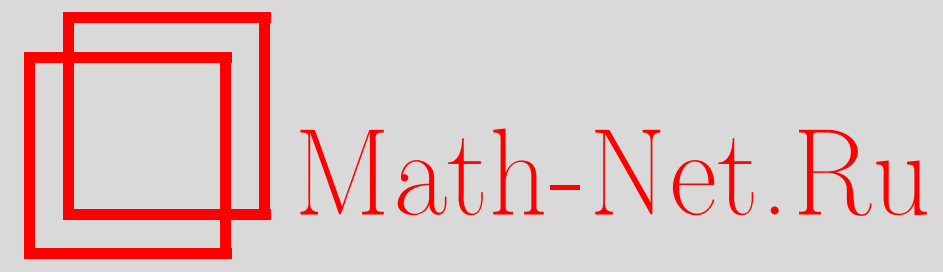

Zh. Su, Normal convergence for random partitions with multiplicative measures, Теория вероятн. и ее примен., 2014, том 59, выпуск 1, 97-129

DOI: https://doi.org/10.4213/tvp4552

Использование Общероссийского математического портала Math-Net.Ru подразумевает, что вы прочитали и согласны с пользовательским соглашением http://www . mathnet.ru/rus/agreement

Параметры загрузки:

IP : 44.207 .124 .84

26 апреля 2023 г., 11:19:52

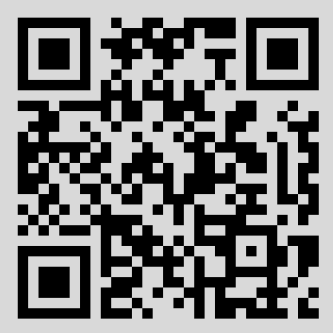




\section{NORMAL CONVERGENCE FOR RANDOM PARTITIONS WITH MULTIPLICATIVE MEASURES}

Пусть $\mathscr{P}_{n}$ - пространство всех разбиений целого числа $n$, $n \geqslant 0, \mathscr{P}$ - пространство всех этих разбиений. Определим на нем класс мультипликативных мер, индуцированных $\mathscr{F}_{\beta}(z)=$ $\prod_{k}\left(1-z^{k}\right)^{k^{\beta}}$, где $\beta>-1$. При помощи предельных форм и других асимптотических свойств, исследованных А. М. Вершиком, в предлагаемой работе установлена нормальная сходимость для объемов и частей случайных разбиений.

Ключевые слова и фразы: центральная предельная теорема, мультипликативная мера, случайные разбиения.

1. Introduction. The problems considered in this paper concern the fluctuations around its limit shape of random partitions of a large integer with respect to multiplicative statistics. Such a study is stimulated by a wide range of applications of random partitions to combinatorics, statistical mechanics, stochastic processes, analytic number theory, etc. The interested reader is referred to [18] and [24] for excellent introduction at this aspect.

Let us start with the precise description of multiplicative statistics introduced first by Vershik [23]. Let $\mathscr{P}_{n}$ be the set of all partitions of integer $n \geqslant 0$ and $\mathscr{P}=\cup_{n} \mathscr{P}_{n}$ the set of all partitions. For $\lambda=\left(\lambda_{1}, \lambda_{2}, \ldots, \lambda_{l}\right) \in \mathscr{P}$, $\lambda_{1} \geqslant \lambda_{2} \geqslant \cdots \geqslant \lambda_{l}>0$, we write $r_{k}(\lambda)=\#\left\{i: \lambda_{i}=k\right\}$ for the number of summands equal to $k$ in the partition $\lambda$, namely $r_{k}(\lambda)$ is the $k$ th occupation number. Obviously, $\left\{r_{k}(\lambda)\right\}$ fully determine the partition $\lambda$; in particular, the size $|\lambda|$ is equal to $\sum_{k} k r_{k}$ and the length $l=\sum_{k} r_{k}$. Now we introduce a class of measures on $\mathscr{P}_{n}$ and $\mathscr{P}$ that are said to be multiplicative. Consider a sequence of functions $f_{k}(z), k \geqslant 1$, analytic in the open disk $D=\{z \in \mathbf{C}:|z|<\varrho\}, \varrho=1$ or $\varrho=\infty$, such that $f_{k}(0)=1$. And assume that

(i) the Taylor series

$$
f_{k}(z)=\sum_{j=0}^{\infty} s_{k}(j) z^{j}
$$

*Department of Mathematics, Zhejiang University, Hangzhou, Zhejiang 310027, P.R. China; e-mail: suzhonggen@zju.eud.cn

1) Supported partly by NSFC grant 11071213, ZJNSF grant R6090034 and Doctoral Fund of Ministry of Education of China grant 20100101110001. 
have all coefficients $s_{k}(j) \geqslant 0$ and

(ii) the infinite product

$$
\mathscr{F}(z)=\prod_{k=1}^{\infty} f_{k}\left(z^{k}\right)
$$

converges in $D$. We can define a family of probability measures $P_{q}, q \in(0, \varrho)$, on $\mathscr{P}$ in the following way: put

$$
P_{q}\left(\lambda \in \mathscr{P}: r_{k}(\lambda)=j\right)=\frac{s_{k}(j) q^{k j}}{f_{k}\left(q^{k}\right)}, \quad j \geqslant 0, \quad k \geqslant 1,
$$

and assume that different occupation numbers are independent. Thus

$$
P_{q}(\lambda)=\frac{\prod_{k=1}^{\infty} s_{k}\left(r_{k}\right)}{\mathscr{F}(q)} q^{|\lambda|}, \quad \lambda \in \mathscr{P} .
$$

Now define the measure $P_{n}$ on $\mathscr{P}_{n}$ by

$$
P_{n}\left(\lambda \in \mathscr{P}_{n}: r_{k}(\lambda)=j\right)=\frac{s_{k}(j)}{Q_{n}}
$$

and

$$
P_{n}(\lambda)=\frac{\prod_{k=1}^{\infty} s_{k}\left(r_{k}\right)}{Q_{n}}, \quad \lambda \in \mathscr{P}_{n},
$$

where

$$
Q_{n}=\sum_{\lambda \vdash n} \prod_{k=1}^{\infty} s_{k}\left(r_{k}\right) .
$$

We remark a basic relation

$$
\mathscr{F}(q)=\sum_{n=0}^{\infty} Q_{n} q^{n}
$$

The following lemma due to [23] describes a key feature of the above measures.

Lemma 1.1. For any $q \in(0, \varrho)$ and $n \geqslant 0$, we have

$$
\left.P_{q}\right|_{\mathscr{P}_{n}}=P_{n},
$$

i.e., $P_{n}$ is the conditional probability measure induced on $\mathscr{P}_{n}$ by $P_{q}$;

$$
P_{q}=\frac{1}{\mathscr{F}(q)} \sum_{n=0}^{\infty} Q_{n} q^{n} P_{n},
$$

i.e., $P_{q}$ is a convex combination of measures $P_{n}$. 
We call the family $\left(\mathscr{P}_{n}, P_{n}\right)$ a small canonical ensemble of partitions and the $\left(\mathscr{P}, P_{q}\right)$ a grand canonical ensemble of partitions in view of similarities to statistical physics. The generating function $\mathscr{F}(z)$, along with its decomposition $\mathscr{F}(z)=\prod_{k=1}^{\infty} f_{k}\left(z^{k}\right)$, completely determines such a family. The above multiplicative measures contain many important examples as discussed in [23] and [26].

For clarity and simplicity, we shall in the sequel restrict our attention to the special case in which the $\mathscr{F}(z)$ is generated by $f_{k}(z)=1 /(1-z)^{k^{\beta}}, \beta>$ -1 . To emphasize the dependence of the ensembles upon the parameter $\beta$, we write $P_{\beta, q}, P_{n, \beta}$ for the probabilities, and $E_{\beta, q}, E_{n, \beta}$ for the mathematical expectations. Also, set

$$
\mathscr{F}_{\beta}(z)=\prod_{k=1}^{\infty} \frac{1}{\left(1-z^{k}\right)^{k^{\beta}}} .
$$

In such a special case, the convergence radius of $f_{k}$ and $\mathscr{F}$ is $\varrho=1$. Vershik [23], Vershik and Yakubovich [26] treat $P_{\beta, q}$ and $P_{n, \beta}$ as generalized Bose-Einstein models of ideal gas; while in combinatorics and number theory they are well known for a long time as weighted partitions.

R e m a r k 1.1. $P_{n, 0}$ corresponds to the uniform measure on $\mathscr{P}_{n}$, and $Q_{n}$ is Euler's function $p(n)$ : the number of partitions of $n$. In the case of $\beta=1$, the $\mathscr{F}_{\beta}(z)$ is the generating function for the numbers $p_{3}(n)$ of 3 -dimensional plane partitions of $n$ :

$$
\sum_{n \geqslant 0} p_{3}(n) z^{n}=\prod_{k \geqslant 1} \frac{1}{\left(1-z^{k}\right)^{k}},
$$

see, e.g., [1]. However, the $P_{n, 1}$ is completely different from the uniform measure on 3-dimensional plane partitions of $n$

To a partition $\lambda \in \mathscr{P}_{n}$ we assign a function $\varphi_{\lambda}$ on $[0, \infty)$ by the following rule:

$$
\varphi_{\lambda}(t)=\varphi_{\lambda}(1), \quad 0 \leqslant t<1
$$

and

$$
\varphi_{\lambda}(t)=\sum_{k=[t]}^{\infty} r_{k}, \quad 1 \leqslant t<\infty,
$$

where $[x]$ denotes the integer part of $x$. Clearly, by definition, $\varphi_{\lambda}(\cdot)$ is a monotone decreasing, piecewise constant function of $t$, and $n=\int_{0}^{\infty} \varphi_{\lambda}(t) d t$. We refer to such a graphical description as a Young diagram of the partition $\lambda$.

Certain asymptotic properties of $P_{n, \beta}$ and $P_{\beta, q}$ have already been well studied in the literature. Vershik [23], in an attempt to capture various limiting results concerning particular functionals in a unified framework, 
posed the question of evaluating the limit shape for $\varphi_{\lambda}(t)$ under $P_{n, \beta}$. For later use, we restate a part of his limit shape results as follows.

Lemma 1.2. Assume $\beta \geqslant 0$, let $h_{n}=(\Gamma(\beta+2) \zeta(\beta+2) / n)^{1 /(\beta+2)}$. Consider the scaled function

$$
\widetilde{\varphi}_{n}(t)=h_{n}^{\beta+1} \varphi_{\lambda}\left(\frac{t}{h_{n}}\right), \quad t \geqslant 0 .
$$

Then we have $\widetilde{\varphi}_{n} \rightarrow \Psi_{\beta}$ in the sense of uniform convergence on compact sets, where $\Psi_{\beta}$ is the function defined by

$$
\Psi_{\beta}(t)=\int_{t}^{\infty} \frac{u^{\beta} e^{-u}}{1-e^{-u}} d u .
$$

More precisely, for any $\varepsilon>0$ and $0<a<b<\infty$, there exists an $n_{0}$ such that for $n>n_{0}$ we have

$$
P_{n, \beta}\left(\lambda \in \mathscr{P}_{n}: \sup _{a \leqslant t \leqslant b}\left|\widetilde{\varphi}_{n}(t)-\Psi_{\beta}(t)\right|>\varepsilon\right)<\varepsilon .
$$

$\mathrm{R}$ e $\mathrm{m}$ a $\mathrm{rk}$ 1.2. The value of $h_{n}$ is in essence determined so that $E_{\beta, q}|\lambda| \sim n$, where $q=e^{-h_{n}}$. For $\beta=0$, the scaling constants along both axes are $\pi n / \sqrt{6}$. Moreover, $\Psi_{0}(t)$ can be written in a more symmetric form

$$
e^{-\pi x / \sqrt{6}}+e^{-\pi y / \sqrt{6}}=1 .
$$

Note also that $\Psi_{0}(t)$ is symmetric about the line $x=y$, and $x=0$ and $y=0$ are its two asymptotic lines, respectively.

For $\beta>0$, two distinct scaling constants must be adapted. In fact, the value on the $y$ axis is more compressed than the indices on the $x$ axis. Also, it is worth noting

$$
\Psi_{\beta}(0)=\int_{0}^{\infty} \frac{u^{\beta} e^{-u}}{1-e^{-u}} d u<\infty
$$

by virtue of $\beta>0$.

Having the limit shape, essentially the law of large numbers, of random partitions, it is natural to ask the question about the asymptotic distribution of fluctuations from the limit curve. Under the probability model $\left(\mathscr{P}, P_{\beta, q}\right)$ with $\beta>-1$, direct calculations can be made to show the limiting distribution of a maximal summand is the Gumbel distribution and analogous results hold for the first $d$ largest summands, see [26]. More specifically, let

$$
A(q)=(\beta+1)|\ln (1-q)|+\beta|\ln (1-q)|+\beta \ln (\beta+1)
$$

and

$$
W_{i}=(1-q) \lambda_{i}-A(q)
$$


Then for any $m \geqslant 1$,

$$
\left(W_{1}, W_{2}, \ldots, W_{m}\right) \stackrel{d}{\longrightarrow}\left(Y_{1}, Y_{2}, \ldots, Y_{m}\right) \quad \text { as } q \rightarrow 1,
$$

where $Y_{1}, Y_{2}, \ldots, Y_{m}$ is a Markov chain such that the density of $Y_{1}$ is $\exp (-x-$ $\left.e^{-x}\right)$ and the density of $Y_{i}$ conditioned on $Y_{i-1}=x$ is $\exp \left(-y-e^{-y}+e^{-x}\right)$, $y \leqslant x$.

The corresponding results for $\left(\mathscr{P}_{n}, P_{n, \beta}\right)$ can be obtained by taking $q=1-e^{-h_{n}}$, where $h_{n}$ is as in Lemma 1.2 and noting the asymptotic equivalence between large and small canonical ensembles. In particular, we have

$$
\lim _{n \rightarrow \infty} P_{n, \beta}\left(\lambda \in \mathscr{P}_{n}: \lambda_{1}-\frac{A_{n}}{h_{n}} \leqslant \frac{t}{h_{n}}\right)=e^{-e^{-t}}
$$

where

$$
A_{n}=\frac{\beta+1}{\beta+2} \ln n+\beta \ln \ln n+\beta \ln \frac{\beta+1}{\beta+2}-\frac{\beta+1}{\beta+2} \ln \Gamma(\beta+2) \zeta(\beta+2) .
$$

The Gumbel limit in (1.4) first appeared in the pioneering work of Erdös and Lehner [7] on uniform random partitions. However, the proof was completely different from that of Vershik and Yakubovich [26]. In fact, using the Hardy-Ramanujan asymptotic formula for $p(n)$, Erdös and Lehner found the limiting distribution of the number $l$ of summands (parts) and so obtained by a classic duality the limiting distribution of the largest summand $\lambda_{1}$.

Remarkably, the classic duality plays a very important role in the asymptotic study of uniform random partitions. Recall that the partition $\lambda^{\prime}$ dual to $\lambda$ is obtained by transposition of $\lambda$. Thus if we write $\left(\lambda_{1}^{\prime}, \ldots, \lambda_{l^{\prime}}^{\prime}\right)$ for $\lambda^{\prime}$, then clearly $\lambda_{1}^{\prime}=l, l^{\prime}=\lambda_{1}$ and there is a simple and useful connection between $\lambda_{j}^{\prime}$ and the sums of $r_{k}(\lambda)$, namely

$$
\lambda_{k}^{\prime}=\sum_{j=k}^{\infty} r_{j}(\lambda), \quad k \geqslant 1
$$

Since $\lambda$ is uniformly random, so is $\lambda^{\prime}$. Therefore, $\left(\lambda_{k}, k \geqslant 1\right) \stackrel{d}{=}\left(\lambda_{k}^{\prime}, k \geqslant 1\right)$.

On the basis of such an elegant duality, Fristedt [10], Pittel [20], [21] undertook a systematic study of the distribution of fluctuations in the bulk of summands for the uniform random partitions that very fruitfully combined analytic and probabilistic tools. The conditioning devices used in their argument is conceptually analogous to the method of equivalence of great and small canonical ensembles above. One of their fundamental results in this aspect can be read as follows: under $\left(\mathscr{P}_{n}, P_{n, 0}\right)$, we have for any $0 \leqslant t<\infty$

$$
h_{n}^{1 / 2}\left(\lambda_{\left[t / h_{n}\right]}-\frac{1}{h_{n}} \Psi_{0}(t)\right) \stackrel{d}{\longrightarrow} N\left(0, \sigma_{0}^{2}(t)\right)
$$


where

$$
\sigma_{0}^{2}(t)=\frac{e^{-t}}{1-e^{-t}}-\frac{3}{\pi^{2}\left(1-e^{-t}\right)^{2}}\left[\left(1-e^{-t}\right) \ln \left(1-e^{-t}\right)+t e^{-t}\right] .
$$

Pittel [20], [21] also discussed a deeper functional central limit theorem and its applications to the character ratio in symmetric group representation and the total number of standard Young tableaux.

Our goal in this paper is to study the distribution of fluctuations from the limit shape under $\left(\mathscr{P}_{n}, P_{n, \beta}\right)$ with $\beta>0$. We shall prove the following analogue of (1.5): for any $0<t<\infty$

$$
h_{n}^{-(\beta+1) / 2}\left(\widetilde{\varphi}_{n}(t)-E_{\beta, q} \widetilde{\varphi}_{n}(t)\right) \stackrel{d}{\longrightarrow} N\left(0, \kappa_{\beta}^{2}(t)\right) .
$$

See Theorem 3.2 in Section 3 for precise statement.

In particular, each $\lambda_{k}^{\prime}, k \geqslant 1$, after properly scaled, is approximately normally distributed when $\beta \geqslant 1$. It is at this point that the fluctuations of the diagram $\lambda$ substantially differ from those in the case of $\beta=0$.

We also remark that the $P_{n, \beta}$ is no longer a uniform measure on $\mathscr{P}_{n}$ when $\beta>0$; so $\lambda$ and $\lambda^{\prime}$ is not necessarily identically distributed. Thus, unfortunately, we cannot use the classic duality to obtain any distribution of fluctuations in the bulk of summands, i.e., $\lambda_{\left[t / h_{n}\right]}$. Still, we used $E_{\beta, q} \widetilde{\varphi}_{n}(t)$ in (1.6) as the centering constant instead of $\Psi_{\beta}(t)$. The reason for this is that $\lambda^{\prime}$ oscillates around its mean more wildly than around its limit curve.

The proof of (1.6) will be given following the line of [20] in Section 3. In fact, by virtue of Lemma 1.1, the probability generating function of $\lambda_{k}^{\prime}$ under $P_{\beta, q}$ is

$$
E_{\beta, q} e^{z \lambda_{k}^{\prime}}=\sum_{n=0}^{\infty} q^{n} Q_{n} E_{n, \beta} e^{z \lambda_{k}^{\prime}}, \quad k \geqslant 1,
$$

from which and using the Cauchy formula, we can express the probability generating function $E_{n, \beta} e^{z \lambda_{k}^{\prime}}$ as an integral of $E_{\beta, q} e^{z \lambda_{k}^{\prime}}$ along a contour around zero. Note that $P_{n, \beta}$ is $P_{\beta, q}$ conditioned on $\mathscr{P}_{n}$ irrespective of the value of $q \in(0,1)$ by virtue of Lemma 1.1 again, so we can choose $q=e^{-h_{n}}$. It remains to calculate the complex integral, in which the saddle point method (equivalently the local limit theorem) is needed. A functional central limit theorem is also proved in Section 3.

Since $P_{\beta, q}$ is a Poissonization of measures $P_{n, \beta}$, the large ensembles must give preponderance to partitions of larger and larger sizes as $q$ grows to 1 . Although needed further confirmation, the asymptotics of the large ensembles as $q$ approaches 1 will often provide a first hint as to what occurs in the small ensembles. As a warm-up, we discuss the distribution of fluctuations for partitions under $\left(\mathscr{P}, P_{\beta, q}\right)$ in Section 2. In this setting, we are in a 
position to deal with the infinite sum of independent and non-identically distributed random variables with negative binomial distributions. Our main result is as follows. Let $q=e^{-h}$ and

$$
V_{h}(t)=h^{(\beta+1) / 2}\left(\lambda_{[t / h]}^{\prime}-E_{\beta, q} \lambda_{[t / h]}^{\prime}\right), \quad t \geqslant 0,
$$

then under $\left(\mathscr{P}, P_{\beta, q}\right)$ with $\beta>1$, we have as $h \rightarrow 0$

$$
V_{h} \Rightarrow V
$$

in the sense of weak convergence in $D[0, \infty)$, where $V=(V(t), t \geqslant 0)$ is a Gaussian process with independent increments.

In Section 4 we shall study the distribution of the total number of standard Young tableaux under $\left(\mathscr{P}_{n}, P_{n, \beta}\right)$. To associated with a partition $\lambda \in \mathscr{P}_{n}$, a standard tableau is obtained by labeling the $n$ cells of the diagram representing $\lambda$ by $1,2, \ldots, n$, so that the labels strictly increase both in columns and rows, in the direction leading away from the diagram's corner. Let $d_{\lambda}$ denote the total number of standard tableaux. Remarkably, the $d_{\lambda}$ is also equal to the degree of the irreducible representation of the symmetric group $S_{n}$ of permutations on $\{1,2, \ldots, n\}$. The Frenbenius formula and the hook formula (see (4.1) and (4.2)) can often be used to effectively compute the $d_{\lambda}$. We shall use the functional central limit theorem obtained in Section 3 to prove that $\ln (n !)^{-1 /(\beta+2)} d_{\lambda}$ suitably scaled is approximately normal distribution when $\beta>1$.

To conclude the Introduction, we remark that the multiplicative measures considered in this paper concern only measures induced by Euler type generating functions. A number of important measures on partitions do not belong to this class. One example is the Plancherel measure much studied and still active in the literature. The so-called Plancherel measure assigns a probability $d_{\lambda}^{2} / n$ ! to a partition $\lambda \in \mathscr{P}_{n}$. In 1977 Vershik and Kerov [25], and independently, Logan and Shepp [16] found the limit shape of a Young diagram with respect to the Plancherel measure. Around 2000 several groups of researchers, Borodin, Okounkov, and Olshanski [5], Johannson [14], Okounkov [17], derived the Tracy-Widom distribution of fluctuations at the end of a Young diagram, while Kerov [15], Ivanov and Olshanski [13] obtained the global Gaussian fluctuation around the limit shape. Bogachev and $\mathrm{Su}[4]$ proved a central limit theorem in the bulk of partitions.

Another interesting example is the multiplicative measure given by the exponential generating function. Let $a=\left(a_{k}, k \geqslant 1\right)$ be a parameter function determined by $g(x)=\exp \left(\sum_{k \geqslant 1} a_{k} x^{k}\right)$. Define a probability $\mu_{n}$ on $\mathscr{P}_{n}$ by

$$
\mu_{n}(\lambda)=\frac{1}{C_{n}} \prod_{k=1} \frac{a_{k}^{r_{k}}}{r_{k} !}, \quad \lambda \in \mathscr{P}_{n},
$$

where $C_{n}$ is the partition function. 
In terms of the form of parameter function, the measure $\mu_{n}$ substantially differ from the $P_{n}$ defined in (1.1). The reader is referred to Erlihson and Granovsky [8] and the reference therein for the limit shape and functional central limit theorem for the fluctuation.

Throughout the paper we denote by $c_{\beta}$ and $C_{\beta}$ numerical constants possibly depending on the parameter $\beta$. They may take different values from line to line.

2. Large canonical ensembles. Recall that $r_{k}(\lambda)=\#\left\{i: \lambda_{i}=k\right\}$ for $\lambda=\left(\lambda_{1}, \cdots, \lambda_{l}\right) \in \mathscr{P}$. Under $P_{\beta, q}$, the $r_{k}$ 's are independent random variables with negative binomial distributions. In particular,

$$
P_{\beta, q}\left(r_{k}=j\right)=\left(1-q^{k}\right)^{k^{\beta}} s_{k, \beta}(j) q^{j k}, \quad j \geqslant 0,
$$

where $s_{k, \beta}(j)$ is such that

$$
\frac{1}{\left(1-q^{k}\right)^{k^{\beta}}}=\sum_{j=0}^{\infty} s_{k, \beta}(j) q^{j} .
$$

In this section we shall use the standard argument for sums of independent random variables to obtain the limiting distributions of $|\lambda|$ and $\lambda_{k}^{\prime}$ of a random partition as $q \rightarrow 1$.

We need the following basic facts on the $r_{k}$ 's:

$$
E_{\beta, q}\left(r_{k}\right)=k^{\beta} \frac{q^{k}}{1-q^{k}}, \quad \operatorname{Var}_{\beta, q}\left(r_{k}\right)=k^{\beta} \frac{q^{k}}{\left(1-q^{k}\right)^{2}}
$$

and

$$
E_{\beta, q} e^{x r_{k}}=\frac{\left(1-q^{k}\right)^{k^{\beta}}}{\left(1-e^{x} q^{k}\right)^{k^{\beta}}} .
$$

Our first result is the following theorem.

Theorem 2.1. Let $q=e^{-h}$.

(1) Under $P_{\beta, q}$ with $\beta>-1$, we have as $h \rightarrow 0$

$$
h^{(\beta+3) / 2}\left(|\lambda|-E_{\beta, q}|\lambda|\right) \stackrel{d}{\longrightarrow} N\left(0, \sigma_{\beta+2}^{2}\right),
$$

where

$$
\sigma_{\beta+2}^{2}=\int_{0}^{\infty} \frac{u^{\beta+2} e^{-u}}{\left(1-e^{-u}\right)^{2}} d u .
$$

(2) Under $P_{-1, q}$, we have as $h \rightarrow 0$

$$
\frac{h}{\sqrt{|\ln h|}}\left(|\lambda|-E_{-1, q}|\lambda|\right) \stackrel{d}{\longrightarrow} N(0,1) .
$$


P r o o f. We shall first give the proof of (2.3), and (2.4) can be similarly proved with a minor modification. Since $|\lambda|=\sum_{k=1}^{\infty} k r_{k}$, then by virtue of (2.1) and (2.2), it is easy to obtain

$$
\begin{gathered}
\mu_{h}=: E_{\beta, q}|\lambda|=\sum_{k=1}^{\infty} k^{\beta+1} \frac{e^{-h k}}{1-e^{-h k}}, \\
\sigma_{h}^{2}=: \operatorname{Var}_{\beta, q}(|\lambda|)=\sum_{k=1}^{\infty} k^{\beta+2} \frac{e^{-h k}}{\left(1-e^{-h k}\right)^{2}},
\end{gathered}
$$

and

$$
E_{\beta, q}\left(e^{x|\lambda|}\right)=\prod_{k=1}^{\infty} \frac{\left(1-e^{-h k}\right)^{k^{\beta}}}{\left(1-e^{x k} e^{-h k}\right)^{k^{\beta}}} .
$$

We shall prove for each $x \in \mathbf{C}$,

$$
E_{\beta, q} e^{x\left(|\lambda|-\mu_{h}\right) / \sigma_{h}}=e^{x^{2} / 2+o(1)},
$$

from which the desired result (2.3) immediately follows. Here and in the sequel the $o(\cdot), O(\cdot)$ and $\sim$ refers to $h \rightarrow 0+$ (equivalently, $q \rightarrow 1-$ ).

To this end, it is sufficient to show

$$
\ln E_{\beta, q} e^{x|\lambda| / \sigma_{h}}=\frac{x \mu_{h}}{\sigma_{h}}+\frac{x^{2}}{2}+o(1) .
$$

It follows from (2.5) that

$$
\begin{aligned}
\ln E_{\beta, q} e^{x|\lambda| / \sigma_{h}} & =\sum_{k=1}^{\infty} k^{\beta} \ln \frac{1-e^{-h k}}{1-e^{x k / \sigma_{h}} e^{-h k}} \\
& =-\sum_{k=1}^{\infty} k^{\beta} \ln \left(1-\frac{\left(e^{x k / \sigma_{h}}-1\right) e^{-h k}}{1-e^{-h k}}\right) .
\end{aligned}
$$

To compute the sum of (2.6), we use the Taylor expansion for the logarithm function $\ln (1-x)$ to yield

$$
\begin{aligned}
\ln \left(1-\frac{\left(e^{x k / \sigma_{h}}-1\right) e^{-h k}}{1-e^{-h k}}\right)= & -\frac{\left(e^{x k / \sigma_{h}}-1\right) e^{-h k}}{1-e^{-h k}}-\frac{\left(e^{x k / \sigma_{h}}-1\right)^{2} e^{-2 h k}}{2\left(1-e^{-h k}\right)^{2}} \\
& +O\left(\frac{\left(e^{x k / \sigma_{h}}-1\right)^{3} e^{-3 h k}}{\left(1-e^{-h k}\right)^{3}}\right)
\end{aligned}
$$

and then for the exponential function $e^{x}$ to yield

$$
\begin{aligned}
\frac{\left(e^{x k / \sigma_{h}}-1\right) e^{-h k}}{1-e^{-h k}}= & \frac{x}{\sigma_{h}} \cdot \frac{k e^{-h k}}{1-e^{-h k}}+\frac{x^{2}}{2 \sigma_{h}^{2}} \cdot \frac{k^{2} e^{-h k}}{1-e^{-h k}} \\
& +O\left(\left(\frac{x}{\sigma_{h}}\right)^{3} \cdot \frac{k^{3} e^{-h k}}{1-e^{-h k}}\right)
\end{aligned}
$$


and

$$
\frac{\left(e^{x k / \sigma_{h}}-1\right)^{2} e^{-2 h k}}{2\left(1-e^{-h k}\right)^{2}}=\frac{x^{2}}{2 \sigma_{h}^{2}} \cdot \frac{k^{2} e^{-2 h k}}{\left(1-e^{-h k}\right)^{2}}+O\left(\left(\frac{x}{\sigma_{h}}\right)^{3} \cdot \frac{k^{3} e^{-2 h k}}{\left(1-e^{-h k}\right)^{2}}\right) .
$$

Substituting (2.7)-(2.9) into (2.6) gives

$$
-\sum_{k=1}^{\infty} k^{\beta} \ln \frac{1-e^{x k / \sigma_{h}} e^{-h k}}{1-e^{-h k}}=\frac{x \mu_{h}}{\sigma_{h}}+\frac{x^{2}}{2}+O\left(\frac{1}{\sigma_{h}^{3}} \sum_{k=1}^{\infty} \frac{k^{\beta+3} e^{-k h}}{\left(1-e^{-k h}\right)^{3}}\right) .
$$

To complete the proof, we need only to verify

$$
\frac{1}{\sigma_{h}^{3}} \sum_{k=1}^{\infty} \frac{k^{\beta+3} e^{-k h}}{\left(1-e^{-k h}\right)^{3}}=O\left(h^{(\beta+1) / 2}\right)=o(1) .
$$

Note that (2.10) explains the usage of the expansion formulae above was indeed legitimate. Now use the approximation of Riemann sums by integral to get as $h \rightarrow 0$

$$
\sigma_{h}^{2}=\sum_{k=1}^{\infty} \frac{k^{\beta+2} e^{-k h}}{\left(1-e^{-k h}\right)^{2}} \sim \frac{1}{h^{\beta+3}} \int_{0}^{\infty} \frac{u^{\beta+2} e^{-u}}{\left(1-e^{-u}\right)^{2}} d u
$$

and

$$
\sum_{k=1}^{\infty} \frac{k^{\beta+3} e^{-k h}}{\left(1-e^{-k h}\right)^{3}} \sim \frac{1}{h^{\beta+4}} \int_{0}^{\infty} \frac{u^{\beta+3} e^{-u}}{\left(1-e^{-u}\right)^{3}} d u .
$$

Thus (2.10) is valid as desired.

When $\beta=-1$, the integrals in the right-hand sides of (2.11) and (2.12) do not exist. However, we can use the Euler-Maclaurin sum formula (see [6]) to estimate the infinite sums in the left-hand sides. In fact, we have

$$
\sigma_{h}^{2}=\sum_{k=1}^{\infty} \frac{k e^{-k h}}{\left(1-e^{-k h}\right)^{2}}=\frac{1}{h^{2}}|\ln h|+o\left(\frac{1}{h^{2}}\right)
$$

and

$$
\sum_{k=1}^{\infty} \frac{k^{2} e^{-k h}}{\left(1-e^{-k h}\right)^{3}}=\frac{1}{h^{3}}|\ln h|+o\left(\frac{1}{h^{3}}\right),
$$

from which (2.10) becomes

$$
\frac{1}{\sigma_{h}^{3}} \sum_{k=1}^{\infty} \frac{k^{2} e^{-k h}}{\left(1-e^{-k h}\right)^{3}}=O\left(\frac{1}{\sqrt{|\ln h|}}\right)=o(1) .
$$

Now (2.4) easily follows. Theorem 2.1 is proved.

$\mathrm{R}$ e $\mathrm{m}$ a $\mathrm{r} \mathrm{k} 2.1$. In the case $\beta=0$, the proof of Theorem 2.1 is contained in [3] and [20].

Analogously, we can obtain the following central limit theorem for $\lambda_{k}^{\prime}$, $k \geqslant 1$. 
Theorem 2.2. (1) Under $P_{\beta, q}$ with $\beta>1$, we have as $h \rightarrow 0$

$$
h^{(\beta+1) / 2}\left(\lambda_{k}^{\prime}-E_{\beta, q} \lambda_{k}^{\prime}\right) \stackrel{d}{\longrightarrow} N\left(0, \sigma_{\beta}^{2}\right),
$$

where

$$
\sigma_{\beta}^{2}=\int_{0}^{\infty} \frac{u^{\beta} e^{-u}}{\left(1-e^{-u}\right)^{2}} d u
$$

(2) Under $P_{1, q}$, we have as $h \rightarrow 0$

$$
\frac{h}{\sqrt{|\ln h|}}\left(\lambda_{k}^{\prime}-E_{1, q} \lambda_{k}^{\prime}\right) \stackrel{d}{\longrightarrow} N(0,1) .
$$

$\mathrm{R}$ e $\mathrm{m}$ a r k 2.2. When $\beta=0$, the central limit theorem obviously fails for $\lambda_{k}^{\prime}, k \geqslant 1$. Indeed, $\lambda_{k}^{\prime}$ asymptotically follows the Gumbel distribution by (1.4) and the duality. Even when $0<\beta<1$, Theorem 2.2 is no longer valid. This is easily understood from the following simple observation: it follows

$$
\sigma_{h, k}^{2}=: \operatorname{Var}_{\beta, q}\left(\lambda_{k}^{\prime}\right)=\sum_{j=k}^{\infty} j^{\beta} \frac{e^{-h j}}{\left(1-e^{-h j}\right)^{2}} \sim \frac{1}{(1-\beta) k^{1-\beta} h^{2}}
$$

and

$$
\sum_{j=k}^{\infty} j^{\beta} \frac{e^{-h j}}{\left(1-e^{-h j}\right)^{3}} \sim \frac{1}{(2-\beta) k^{2-\beta} h^{3}}
$$

and so

$$
\frac{1}{\sigma_{h, k}^{3}} \sum_{j=k}^{\infty} \frac{j^{\beta} e^{-j h}}{\left(1-e^{-j h}\right)^{3}} \sim \frac{(1-\beta)^{3 / 2}}{(2-\beta) k^{(\beta+1) / 2}}>0 .
$$

Further, we turn to the distribution of fluctuations in the bulk of a partition. Define

$$
V_{h}(t)=h^{(\beta+1) / 2}\left(\lambda_{[t / h]}^{\prime}-E_{\beta, q} \lambda_{[t / h]}^{\prime}\right), \quad t \geqslant 0,
$$

where by convention $\lambda_{0}^{\prime}=\lambda_{1}^{\prime}$.

Theorem 2.3. Under $\left(\mathscr{P}, P_{\beta, q}\right)$ with $\beta>-1$, we have as $h \rightarrow 0$

(1) for each $t>0$

$$
V_{h}(t) \stackrel{d}{\longrightarrow} N\left(0, \sigma_{\beta}^{2}(t)\right),
$$

where

$$
\sigma_{\beta}^{2}(t)=\int_{t}^{\infty} \frac{u^{\beta} e^{-u}}{\left(1-e^{-u}\right)^{2}} d u
$$


(2) for any $a>0$

$$
V_{h} \Rightarrow G \quad \text { in } D[a, \infty),
$$

where $G$ is a continuous Gaussian process with independent increments.

$\mathrm{P}$ r o o f. Convergence (2.14) can be proved in a completely similar argument to (2.3). We need only to prove (2.15).

Since $V_{h}$ is a partial sum process of independent random variables, we can apply the general theory of weak convergence in $D[a, \infty)$ (see [2]). From (2.14) and the independence of the $r_{k}$ 's one can easily see the convergence of a finite dimensional distribution of $V_{h}$. Therefore, it is sufficient to establish the uniform tightness for $V_{h}$ in $D[a, \infty)$. In turn, it is enough to show that for each positive $\varepsilon$ and $\eta$, there exist a $\delta, 0<\delta<1$, and an $h_{0}>0$ such that for any $h<h_{0}$,

$$
P_{\beta, q}\left(\sup _{|t-s| \leqslant \delta}\left|V_{h}(s)-V_{h}(t)\right|>\eta\right)<\varepsilon .
$$

Let $S_{k}=\lambda_{k}^{\prime}-E_{\beta, q} \lambda_{k}^{\prime}$. Then according to (7.12) of [2], a sufficient condition for (2.16) to hold is for every $t \geqslant a$

$$
\frac{1}{\delta} P_{\beta, q}\left(\max _{[t / h] \leqslant k \leqslant[(t+\delta) / h]}\left|S_{[t / h]}-S_{k}\right|>\frac{\eta}{h^{(\beta+1) / 2}}\right)<\varepsilon .
$$

To this end, we use Lévy's inequality (see Theorem 12 of [19, Chap. III]) to get

$$
\begin{aligned}
& P_{\beta, q}\left(\max _{[t / h] \leqslant k \leqslant[(t+\delta) / h]}\left|S_{[t / h]}-S_{k}\right|>\frac{\eta}{h^{(\beta+1) / 2}}\right) \\
& \leqslant 2 P_{\beta, q}\left(\left|S_{[t / h]}-S_{[(t+\delta) / h]}\right|>\frac{\eta}{h^{(\beta+1) / 2}}-\sqrt{2 \operatorname{Var}_{\beta, q}\left(S_{[t / h]}-S_{[(t+\delta) / h]}\right)}\right)
\end{aligned}
$$

Choose $\delta$ small enough (not depending on $t$ and $h$ ) that

$$
\operatorname{Var}_{\beta, q}\left(S_{[t / h]}-S_{[(t+\delta) / h]}\right) \leqslant \frac{C_{\beta}}{h^{\beta+1}} \int_{t}^{t+\delta} \frac{u^{\beta} e^{-u}}{\left(1-e^{-u}\right)^{2}} d u<\frac{\eta^{2}}{8 h^{\beta+1}} .
$$

Then the probability in the right-hand side of (2.18) is bounded by

$$
P_{\beta, q}\left(\left|S_{[t / h]}-S_{[(t+\delta) / h]}\right|>\frac{\eta}{2 h^{(\beta+1) / 2}}\right) .
$$

Further, we shall use Chebyschev's inequality to further control (2.19) from above. Note that

$$
E_{\beta, q} r_{k}^{3}=k^{\beta}\left(k^{\beta}+1\right)\left(k^{\beta}+2\right) \frac{q^{3 k}}{\left(1-q^{k}\right)^{3}}+3 E_{\beta, q} r_{k}^{2}-2 E_{\beta, q} r_{k}
$$


and

$$
\begin{aligned}
E_{\beta, q} r_{k}^{4}= & k^{\beta}\left(k^{\beta}+1\right)\left(k^{\beta}+2\right)\left(k^{\beta}+3\right) \frac{q^{4 k}}{\left(1-q^{k}\right)^{4}} \\
& +6 E_{\beta, q} r_{k}^{3}-11 E_{\beta, q} r_{k}^{2}+6 E_{\beta, q} r_{k}
\end{aligned}
$$

These together with (2.1) leads to

$$
\begin{aligned}
E_{\beta, q}\left(r_{k}-E_{\beta, q} r_{k}\right)^{4} \\
=E_{\beta, q} r_{k}^{4}-4 E_{\beta, q} r_{k}^{3}\left(E_{\beta, q} r_{k}\right)+6 E_{\beta, q} r_{k}^{2}\left(E_{\beta, q} r_{k}\right)^{2}-3\left(E_{\beta, q} r_{k}\right)^{4} \\
=k^{2 \beta}\left[\frac{11 e^{-4 u}-4 e^{-3 u}-4 e^{-2 u}}{\left(1-e^{-u}\right)^{4}}+\frac{18 e^{-2 u}}{\left(1-e^{-u}\right)^{3}}-\frac{11 e^{-2 u}}{\left(1-e^{-u}\right)^{2}}\right] \\
\quad+k^{\beta}\left[\frac{6 e^{-4 u}}{\left(1-e^{-u}\right)^{4}}+\frac{12 e^{-2 u}}{\left(1-e^{-u}\right)^{3}}-\frac{5 e^{-u}}{\left(1-e^{-u}\right)^{2}}+\frac{6 e^{-u}}{\left(1-e^{-u}\right)}\right] \\
=: k^{2 \beta} g_{1}(u)+k^{\beta} g_{2}(u),
\end{aligned}
$$

where $e^{-u}=q^{k}$.

Using the independence of $r_{k}$ 's we have

$$
\begin{aligned}
E_{\beta, q}\left|S_{[t / h]}-S_{[(t+\delta) / h]}\right|^{4} \leqslant & \sum_{k=[t / h]}^{[(t+\delta) / h]} E_{\beta, q}\left(r_{k}-E r_{k}\right)^{4} \\
& +3\left(\sum_{k=[t / h]}^{[(t+\delta) / h]} \operatorname{Var}_{\beta, q}\left(r_{k}\right)\right)^{2}
\end{aligned}
$$

Thus by Chebyschev's inequality and (2.20) we obtain

$$
\begin{gathered}
P_{\beta, q}\left(\left|S_{[t / h]}-S_{[(t+\delta) / h]}\right|>\frac{\eta}{2 h^{(\beta+1) / 2}}\right) \\
\leqslant \frac{2^{4} h^{2(\beta+1)}}{\eta^{4}}\left[\sum_{k=[t / h]}^{[(t+\delta) / h]} E_{\beta, q}\left(r_{k}-E r_{k}\right)^{4}+3\left(\sum_{k=[t / h]}^{[(t+\delta) / h]} \operatorname{Var}_{\beta, q}\left(r_{k}\right)\right)^{2}\right] \\
\leqslant \frac{C_{\beta} h^{2(\beta+1)}}{\eta^{4}}\left[\frac{1}{h^{2 \beta+1}} \int_{t}^{t+\delta} u^{2 \beta} g_{1}(u) d u+\frac{1}{h^{\beta+1}} \int_{t}^{t+\delta} u^{\beta} g_{2}(u) d u\right. \\
\left.\quad+\frac{3}{h^{2(\beta+1)}}\left(\int_{t}^{t+\delta} \frac{u^{\beta} e^{-u}}{\left(1-e^{-u}\right)^{2}} d u\right)^{2}\right] \\
=\frac{C_{\beta}}{\eta^{4}}\left[h \int_{t}^{t+\delta} u^{2 \beta} g_{1}(u) d u+h^{\beta+1} \int_{t}^{t+\delta} u^{\beta} g_{2}(u) d u\right. \\
\left.\quad+3\left(\int_{t}^{t+\delta} \frac{u^{\beta} e^{-u}}{\left(1-e^{-u}\right)^{2}} d u\right)^{2}\right] .
\end{gathered}
$$

Note

$$
\sup _{a \leqslant u<\infty} u^{2 \beta} g_{1}(u)<\infty, \quad \sup _{a \leqslant u<\infty} u^{2 \beta} g_{2}(u)<\infty, \quad \frac{u^{\beta} e^{-u}}{\left(1-e^{-u}\right)^{2}}<\infty .
$$


Hence we have

$$
P_{\beta, q}\left(\left|S_{[t / h]}-S_{[(t+\delta) / h]}\right|>\frac{\eta}{2 h^{(\beta+1) / 2}}\right) \leqslant \frac{C_{\beta}}{\eta^{4}}\left(h+h^{\beta+1}+\delta\right) \delta,
$$

which immediately implies (2.17) holds as long as we choose $\delta$ and $\eta$ sufficiently small. This completes the proof of Theorem 2.3 as desired.

$\mathrm{R}$ e $\mathrm{m}$ a r k 2.3. As we have already seen in Lemma 1.2, the curve $\Psi_{\beta}(t)$ is the limit shape for random partitions under $\left(\mathscr{P}, P_{\beta, q}\right)$. But we cannot in general replace the centering constant $E_{\beta, q} \lambda_{[t / h]}^{\prime}$ by $\Psi_{\beta}(t) / h^{\beta+1}$ in (2.13). In fact, let

$$
f(x)=\frac{x^{\beta} e^{-x}}{1-e^{-x}},
$$

then by the Euler-Maclaurin sum formula, we have for $t>0$ and $m \geqslant 1$

$$
\begin{aligned}
E_{\beta, q} \lambda_{[t / h]}^{\prime} & =\sum_{k=[t / h]}^{\infty} \frac{k^{\beta} e^{-k h}}{1-e^{-k h}}=\frac{1}{h^{\beta}} \sum_{k=[t / h]}^{\infty} f(h k) \\
=\frac{1}{h^{\beta}}[ & \int_{[t / h]}^{\infty} f(h x) d x+\frac{1}{2} f\left(h\left[\frac{t}{h}\right]\right)-\sum_{l=1}^{m} \frac{B_{2 l}}{(2 l) !} h^{2 l-1} f^{(2 l-1)}\left(h\left[\frac{t}{h}\right]\right) \\
& \left.\quad-h^{2 m} \int_{[t / h]}^{\infty} f^{(2 m)}(h x) \frac{B_{2 m}(x-[x])}{(2 m) !} d x\right],
\end{aligned}
$$

where $B_{l}(x)$ denotes the $l$ th Bernoulli polynomials.

It is easy to see that

$$
\int_{\left[\frac{t}{h}\right]}^{\infty} f(h x) d x=\frac{1}{h} \Psi_{\beta}(t)-\frac{1}{h} \int_{t}^{h[t / h]} f(x) d x .
$$

Also, since $\left|B_{2 m}(x-[x])\right| \leqslant\left|B_{2 m}\right|<\infty$, it follows that

$$
\begin{aligned}
\left|\int_{[t / h]}^{\infty} f^{(2 m)}(h x) \frac{B_{2 m}(x-[x])}{(2 m) !} d x\right| & \leqslant \frac{\left|B_{2 m}\right|}{(2 m) !} \int_{[t / h]}^{\infty}\left|f^{(2 m)}(h x)\right| d x \\
& \leqslant \frac{\left|B_{2 m}\right|}{h(2 m) !} \int_{t}^{\infty}\left|f^{(2 m)}(x)\right| d x .
\end{aligned}
$$

When $\beta=0$, we have for $t>0$

$$
E_{0, q} \lambda_{[t / h]}^{\prime}=\frac{1}{h} \Psi_{0}(t)+O(1),
$$

which implies (2.14) is still valid with $\Psi_{0}(t) / t$ in place of $E_{0, q} \lambda_{[t / h]}^{\prime}$ in (2.13).

3. Small canonical ensembles. Let $\sigma_{n}^{2}=h_{n}^{-(\beta+1)}$ and define for $k \geqslant 1$

$$
\mu_{n, k}=\sum_{j=k}^{\infty} j^{\beta} \frac{e^{-h_{n} j}}{1-e^{-h_{n} j}}, \quad \sigma_{n, k}^{2}=\sum_{j=k}^{\infty} j^{\beta} \frac{e^{-h_{n} j}}{\left(1-e^{-h_{n} j}\right)^{2}},
$$

where $h_{n}$ is as in Lemma 1.2. 
Theorem 3.1. (1) Under $P_{n, \beta}$ with $\beta>1$, we have as $n \longrightarrow \infty$,

$$
\frac{\lambda_{k}^{\prime}-\mu_{n, k}}{\sigma_{n}} \stackrel{d}{\longrightarrow} N\left(0, \kappa_{\beta}^{2}(0)\right)
$$

where

$$
\kappa_{\beta}^{2}(0)=\Gamma(\beta+1) \zeta(\beta+1,0)-\frac{\Gamma(\beta+2) \zeta^{2}(\beta+2,0)}{\zeta(\beta+2)}
$$

and

$$
\zeta(r+1,0)=: \frac{1}{\Gamma(r+1)} \int_{0}^{\infty} \frac{u^{r} e^{-u}}{\left(1-e^{-u}\right)^{2}} d u \quad \text { for } r>1 .
$$

(2) Under $P_{n, 1}$, we have as $n \rightarrow \infty$,

$$
\frac{\lambda_{k}^{\prime}-\mu_{n, k}}{\sigma_{n} \sqrt{\left|\ln h_{n}\right|}} \stackrel{d}{\longrightarrow} N(0,1) .
$$

$\mathrm{P} r$ o o f. The proof basically follows the line of Theorem 5 of [20]. We shall only give the proof of (3.2), and (3.3) can be similarly proved with some minor modifications.

It suffices to verify for each $u \in \mathbf{R}$

$$
E_{n, \beta} \exp \left(u \frac{\lambda_{k}^{\prime}-\mu_{n, k}}{\sigma_{n}}\right) \longrightarrow \exp \left(\frac{1}{2} \kappa_{\beta}^{2}(0) u^{2}\right) .
$$

Recall the generating function of $\lambda_{k}^{\prime}$ under $P_{\beta, q}$ is

$$
E_{\beta, q} e^{x \lambda_{k}^{\prime}}=\prod_{j=k}^{\infty} \frac{\left(1-q^{j}\right)^{j^{\beta}}}{\left(1-e^{x} q^{j}\right)^{j^{\beta}}} .
$$

So by Lemma 1.1, we have

$$
\sum_{m=0}^{\infty} q^{m} Q_{m} E_{m, \beta} e^{x \lambda_{k}^{\prime}}=\mathscr{F}_{\beta}(q) E_{\beta, q} e^{x \lambda_{k}^{\prime}}=\mathscr{F}_{\beta}(q) \prod_{j=k}^{\infty} \frac{\left(1-q^{j}\right)^{j^{\beta}}}{\left(1-e^{x} q^{j}\right)^{j^{\beta}}} .
$$

We view the right-hand side of above relation as an analytic function in $\{q \in \mathbf{C}:|q|<1\}$, and make use of the Cauchy integral formula to get

$$
E_{n, \beta} e^{x \lambda_{k}^{\prime}}=\frac{1}{2 \pi Q_{n} \gamma^{n}} \int_{-\pi}^{\pi} e^{-i n \theta} \mathscr{F}_{\beta}\left(\gamma e^{i \theta}\right) \prod_{j=k}^{\infty} \frac{\left(1-\left(\gamma e^{i \theta}\right)^{j}\right)^{j^{\beta}}}{\left(1-e^{x}\left(\gamma e^{i \theta}\right)^{j}\right)^{j^{\beta}}} d \theta .
$$

We are now in a position to choose a suitable radius $\gamma$ and to estimate the complex contour integral in (3.5). For simplicity, define

$$
F_{n}\left(x, \gamma e^{i \theta}\right)=\mathscr{F}_{\beta}\left(\gamma e^{i \theta}\right) \prod_{j=k}^{\infty} \frac{\left(1-\left(\gamma e^{i \theta}\right)^{j}\right)^{j^{\beta}}}{\left(1-e^{x}\left(\gamma e^{i \theta}\right)^{j}\right)^{j^{\beta}}} .
$$


Let $\gamma=e^{-\tau}, \tau>0$ is to be determined (see (3.9) in what follows). We need the following lemma to estimate the generating function $\mathscr{F}_{\beta}\left(\gamma e^{i \theta}\right)$ and the partition function $Q_{n}$. The reader is referred to Lemma 6.1 and Theorem 6.2 of Chapter 6 in [1] for more detail.

Lemma 3.1. Let $\mathscr{F}_{\beta}$ and $Q_{n}$ be as in the Introduction, then

(i) as $\tau \rightarrow 0$

$\mathscr{F}_{\beta}\left(e^{-\tau+i \theta}\right)=\exp \left(\Gamma(\beta+1) \zeta(\beta+2) \tau^{-(\beta+1)}-D(0) \ln \tau+D^{\prime}(0)+O\left(\tau^{A_{0}}\right)\right)$

uniformly in $\theta$ provided $|\theta| \leqslant \pi / 4$, where $A_{0}\left(0<A_{0}<1\right)$ is a constant and

$$
D(s)=\sum_{n=1}^{\infty} \frac{1}{n^{s-\beta}}, \quad s=x+i y,
$$

possesses an analytic continuation in the region $x \geqslant-A_{0}$.

(ii) as $n \rightarrow \infty$

$$
\begin{aligned}
Q_{n}= & A_{1} n^{(2 D(0)-(\beta+3)) /(2(\beta+2))} \\
& \times \exp \left(\frac{\beta+2}{\beta+1}(\Gamma(\beta+2) \zeta(\beta+2))^{1 / \beta+2} n^{(\beta+1) /(\beta+2)}\right)(1+o(1)),
\end{aligned}
$$

where

$$
A_{1}=\frac{e^{D^{\prime}(0)}}{\sqrt{2 \pi(\beta+2)}}(\Gamma(\beta+2) \zeta(\beta+2))^{(1-2 D(0)) /(2(\beta+2))} .
$$

We proceed to prove Theorem 3.1. In view of Lemma 3.1, letting $\theta=0$ in (3.6) yields

$$
\gamma^{-n} F_{n}(x, \gamma)=e^{n \tau} \mathscr{F}_{\beta}\left(e^{-\tau}\right) \prod_{j=k}^{\infty} \frac{\left(1-e^{-j \tau}\right)^{j^{\beta}}}{\left(1-e^{x} e^{-j \tau}\right)^{j^{\beta}}}
$$

Define

$$
H(t, x)=n t+\Gamma(\beta+1) \zeta(\beta+2) t^{-(\beta+1)}+\sum_{j=k}^{\infty} j^{\beta} \ln \frac{1-e^{-j t}}{1-e^{x} e^{-j t}} .
$$

Then substituting (3.7) with $\theta=0$ into (3.7) gives

$$
\gamma^{-n} F_{n}(x, \gamma)=\exp \left(H(\tau, x)-D(0) \ln \tau+D^{\prime}(0)+O\left(\tau^{A_{0}}\right)\right) .
$$

To estimate the value of $H$ at $\tau$, we need its first three derivatives:

$$
\begin{aligned}
H_{t}(t, x)= & n-\Gamma(\beta+2) \zeta(\beta+2) t^{-(\beta+2)} \\
& +\sum_{j=k}^{\infty} j^{\beta+1}\left[\frac{e^{-j t}}{1-e^{-j t}}-\frac{e^{x} e^{-j t}}{1-e^{x} e^{-j t}}\right], \\
H_{t t}(t, x)= & \Gamma(\beta+3) \zeta(\beta+2) t^{-(\beta+3)} \\
& -\sum_{j=k}^{\infty} j^{\beta+2}\left[\frac{e^{-j t}}{\left(1-e^{-j t}\right)^{2}}-\frac{e^{x} e^{-j t}}{\left(1-e^{x} e^{-j t}\right)^{2}}\right],
\end{aligned}
$$


and

$$
\begin{aligned}
H_{t t t}(t, x)= & -\Gamma(\beta+4) \zeta(\beta+2) t^{-(\beta+4)} \\
& -\sum_{j=k}^{\infty} j^{\beta+3}\left[\frac{e^{-j t}\left(1+e^{-j t}\right)}{\left(1-e^{-j t}\right)^{3}}-\frac{e^{x} e^{-j t}\left(1+e^{x} e^{-j t}\right)}{\left(1-e^{x} e^{-j t}\right)^{3}}\right]
\end{aligned}
$$

Now define $\tau$ as follows:

$$
\tau=\tau^{*}\left(1-\frac{1-e^{x}}{(\beta+2) n} S\left(\tau^{*}, x\right)\right)
$$

where

$$
\tau^{*}=h_{n}, \quad S(t, x)=\sum_{j=k}^{\infty} \frac{j^{\beta+1} e^{-j t}}{\left(1-e^{-j t}\right)\left(1-e^{x} e^{-j t}\right)} .
$$

It is easy to see that

$$
H\left(\tau^{*}, x\right)=\frac{(\beta+2) \Gamma(\beta+2) \zeta(\beta+2)}{(\beta+1)\left(\tau^{*}\right)^{\beta+1}}+\sum_{j=k}^{\infty} j^{\beta} \ln \frac{1-e^{-j \tau^{*}}}{1-e^{x} e^{-j \tau^{*}}} .
$$

Let $x=u / \sigma_{n}$. Then as in the proof of Theorem 2.1, we have

$$
\sum_{j=k}^{\infty} j^{\beta} \ln \frac{1-e^{-j \tau^{*}}}{1-e^{x} e^{-j \tau^{*}}}=\frac{\mu_{n, k}}{\sigma_{n}} u+\frac{u^{2}}{2} \Gamma(\beta+1) \zeta(\beta+1,0)+o(1),
$$

where we use the following limits: for $\beta>1$

$$
\frac{1}{\sigma_{n}^{2}} \sum_{j=k}^{\infty} j^{\beta} \frac{e^{-j \tau^{*}}}{\left(1-e^{-j \tau^{*}}\right)^{2}} \longrightarrow \Gamma(\beta+1) \zeta(\beta+1,0)
$$

and

$$
\frac{1}{\sigma_{n}^{3}} \sum_{j=k}^{\infty} j^{\beta} \frac{e^{-j \tau^{*}}}{\left(1-e^{-j \tau^{*}}\right)^{3}} \longrightarrow 0
$$

To estimate $H_{t}(t, x)$ at $t=\tau$, we note

$$
H_{t}(\tau, x)=n-n\left(1-\frac{1-e^{x}}{(\beta+2) n} S\left(\tau^{*}, x\right)\right)^{-(\beta+2)}+\left(1-e^{x}\right) S(\tau, x) .
$$

Making use of the Taylor expansion yields

$$
\begin{aligned}
& \left(1-\frac{1-e^{x}}{n(\beta+2)} S\left(\tau^{*}, x\right)\right)^{-(\beta+2)} \\
& \quad=1+\frac{1-e^{x}}{n} S\left(\tau^{*}, x\right)+\frac{(\beta+3)\left(1-e^{x}\right)^{2}}{2 n^{2}(\beta+2)} S\left(\tau^{*}, x\right)^{2}+O\left(\frac{1}{\sigma_{n}^{2}}\right)
\end{aligned}
$$


and

$$
S(\tau, x)=S\left(\tau^{*}, x\right)-S_{t}\left(\tau^{*}, x\right) \frac{\tau^{*}\left(1-e^{x}\right)}{n(\beta+2)} S\left(\tau^{*}, x\right)+O\left(\frac{n}{\sigma_{n}^{2}}\right) .
$$

Inserting (3.12) and (3.13) into (3.11) we obtain

$$
H_{t}(\tau, x)=O\left(\frac{n}{\sigma_{n}^{2}}\right)=O\left(n^{1 /(\beta+2)}\right) .
$$

Analogously, for $\tau^{*} \leqslant \tilde{t} \leqslant \tau$,

$$
H_{t t}(\tilde{t}, x)=\Gamma(\beta+3) \zeta(\beta+2)\left(\tau^{*}\right)^{-(\beta+3)}+O\left(n^{(\beta+5) / 2(\beta+2)}\right) .
$$

Again, by the Taylor expansion of the function $H(t, x)$ at the point $t=\tau$, we have

$$
H\left(\tau^{*}, x\right)=H(\tau, x)+H_{t}(\tau, x)\left(\tau^{*}-\tau\right)+\frac{1}{2} H_{t t}(\tilde{t}, x)\left(\tau^{*}-\tau\right)^{2}
$$

which in turn, together with (3.14), implies

$$
\begin{aligned}
H(\tau, x) & =H\left(\tau^{*}, x\right)+H_{t}(\tau, x)\left(\tau-\tau^{*}\right)-\frac{1}{2} H_{t t}(\tilde{t}, x)\left(\tau-\tau^{*}\right)^{2} \\
& =\frac{(\beta+2) \Gamma(\beta+2) \zeta(\beta+2)}{(\beta+1)\left(\tau^{*}\right)^{\beta+1}}+\frac{\mu_{n, k}}{\sigma_{n}} u+\frac{u^{2}}{2} \kappa_{\beta}^{2}(0)+o(1) .
\end{aligned}
$$

Substituting (3.16) into (3.8) and by the definition (3.9) of $\tau$, we obtain

$$
\begin{aligned}
& \gamma^{-n} F_{n}(x, \gamma)=\exp \left(\frac{u \mu_{n, k}}{\sigma_{n}}+\frac{u^{2} \kappa_{\beta}^{2}(0)}{2}\right) \frac{n^{D(0) /(\beta+2)}}{(\Gamma(\beta+2) \zeta(\beta+2))^{D(0) /(\beta+2)}} \\
& \quad \times \exp \left(\frac{(\beta+2) \Gamma(\beta+2) \zeta(\beta+2)}{(\beta+1)\left(\tau^{*}\right)^{\beta+1}}+D^{\prime}(0)+O\left(\tau^{A_{0}}\right)\right)(1+o(1)) .
\end{aligned}
$$

Let us turn to the general case $\theta \in(-\pi, \pi)$ and $\gamma=e^{-\tau}$. Applying Lemma 3.1 to (3.6), and noting the definition of $H(t, x)$, we have

$$
\begin{aligned}
& \gamma^{-n} e^{-i n \theta} F_{n}\left(x, \gamma e^{i \theta}\right) \\
& \quad=\exp \left(H(\tau-i \theta, x)-D(0) \ln \tau+D^{\prime}(0)+O\left(\tau^{A_{0}}\right)+O\left(\frac{\theta}{\tau}\right)\right)
\end{aligned}
$$

It similarly follows that

$$
H_{t t t}(\tau, x)=O\left(\frac{1}{\left(\tau^{*}\right)^{\beta+4}}\right) .
$$

Thus we make use of the Taylor expansion of $H(\tau-i \theta, x)$ in $\theta=0$ to get

$$
H(\tau-i \theta, x)=H(\tau, x)-i \theta H_{t}(\tau, x)-\frac{\theta^{2}}{2} H_{t t}(\tau, x)+O\left(\frac{\theta^{3}}{\left(\tau^{*}\right)^{\beta+4}}\right),
$$


which together with (3.8) and (3.17) yields

$$
\begin{aligned}
\gamma^{-n} e^{-i n \theta} F_{n}\left(x, \gamma e^{i \theta}\right)=\gamma^{-n} F_{n}(x, \gamma) \exp ( & -i \theta H_{t}(\tau, x)-\frac{\theta^{2}}{2} H_{t t}(\tau, x) \\
& \left.+O\left(\frac{\theta^{3}}{\left(\tau^{*}\right)^{\beta+4}}\right)+O\left(\frac{\theta}{\tau}\right)\right) .
\end{aligned}
$$

We are now ready to calculate the contour integral (3.5). Let $\theta_{n}=$ $h_{n}^{(\beta+3) / 2} \ln n$. The integral will be split into two parts: $\left\{\theta:|\theta| \leqslant \theta_{n}\right\}$ and $\left\{\theta:|\theta|>\theta_{n}\right\}$.

A direct calculation shows that

$$
\begin{aligned}
\frac{1}{2 \pi} \int_{|\theta| \leqslant \theta_{n}} e^{-i \theta H_{t}(\tau, x)-\theta^{2} H_{t t}(\tau, x) / 2} d \theta=\frac{1}{\sqrt{2 \pi H_{t t}(\tau, x)}} \exp \left(-\frac{H_{t}^{2}(\tau, x)}{2 H_{t t}(\tau, x)}\right) \\
+O\left(H_{t t}^{-1 / 2} \exp \left(-\frac{1}{2} \theta_{n}^{2} H_{t t}(\tau, x)\right)\right) .
\end{aligned}
$$

Since

$$
\frac{H_{t}^{2}(\tau, x)}{H_{t t}(\tau, x)}=O\left(n^{-(\beta+1) /(\beta+2)}\right)
$$

and $\theta_{n}^{2} H_{t t}(\tau, x) \geqslant a \ln ^{2} n$, for some $a>0$, then we obtain

$$
\begin{gathered}
\frac{1}{2 \pi \gamma^{n}} \int_{|\theta| \leqslant \theta_{n}} e^{-i n \theta} F_{n}\left(x, \gamma e^{i \theta}\right) d \theta=\frac{\gamma^{-n} F_{n}(x, \gamma)\left(\tau^{*}\right)^{(\beta+3) / 2}}{\sqrt{2 \pi \Gamma(\beta+3) \zeta(\beta+2)}}(1+o(1)) \\
=Q_{n} \exp \left(\frac{u \mu_{n, k}}{\sigma_{n}}+\frac{u^{2} \kappa_{\beta}^{2}(0)}{2}\right)(1+o(1)) .
\end{gathered}
$$

To estimate the integral over the region $\left\{\theta:|\theta|>\theta_{n}\right\}$, observe that it follows by an elementary inequality due to (1.11) of [20],

$$
\begin{aligned}
\left|F_{n}\left(x, \gamma e^{i \theta}\right)\right| & \leqslant \prod_{j=1}^{\infty} \frac{1}{\left(1-\gamma^{j}\right)^{j^{\beta}}} \prod_{j=k}^{\infty} \frac{1-\gamma^{j}}{1-e^{x} \gamma^{j}} \exp \left(\sum_{j=1}^{\infty} j^{\beta} \gamma^{j}(\cos j \theta-1)\right) \\
& =F_{n}(x, \gamma) \exp \left(\sum_{j=1}^{\infty} j^{\beta} \gamma^{j}(\cos j \theta-1)\right) .
\end{aligned}
$$

We need to calculate the infinite sum in the exponential of (3.19). In a similar argument to (1.9) of [20], one can prove that there is a positive constant $A_{3}$ such that if $|\theta| \leqslant A_{3} h_{n}$, then

$$
\sum_{j=1}^{\infty} j^{\beta} \gamma^{j}(1-\cos j \theta) \geqslant \frac{c_{\beta} \theta^{2}}{h_{n}^{\beta+3}} .
$$

On the other hand, if $|\theta| \geqslant A_{3} h_{n}$, then by the Euler-Maclaurin formula

$$
\sum_{j=1}^{\infty} j^{\beta} \gamma^{j}(1-\cos j \theta)=\sum_{j=1}^{\infty} j^{\beta} e^{-j \tau}(1-\cos j \theta)
$$




$$
\begin{aligned}
& =\frac{1}{\tau^{\beta+1}} \int_{0}^{\infty} u^{\beta} e^{-u}\left(1-\cos \frac{\theta}{\tau} u\right) d u+O\left(\frac{1}{\tau^{\beta}}\right) \\
& \geqslant \frac{c_{\beta}}{h_{n}^{\beta+1}},
\end{aligned}
$$

where in the last inequality we used (3.9).

By (3.19), (3.20) and noting $\theta_{n}=h_{n}^{(\beta+3) / 2} \ln n$, it easily follows

$$
\begin{aligned}
\int_{|\theta| \geqslant \theta_{n}} & \exp \left(\sum_{j=1}^{\infty} j^{\beta} \gamma^{j}(\cos j \theta-1)\right) d \theta \\
& \leqslant \int_{\theta_{n} \leqslant|\theta| \leqslant A_{3} h_{n}} \exp \left(-\frac{c_{\beta} \theta^{2}}{h_{n}^{\beta+3}}\right) d \theta+\int_{|\theta| \geqslant A_{3} h_{n}} \exp \left(-\frac{c_{\beta}}{h_{n}^{\beta+1}}\right) d \theta \\
& \leqslant 2 \exp \left(-c_{\beta} \ln ^{2} n\right) .
\end{aligned}
$$

Thus by (3.19) and (3.22) we have

$$
\left|\frac{1}{2 \gamma^{n}} \int_{|\theta| \geqslant \theta_{n}} e^{-i n \theta} F_{n}\left(x, \gamma e^{i \theta}\right) d \theta\right| \leqslant \gamma^{-n} F_{n}(x, \gamma) \exp \left(-c_{\beta} \ln ^{2} n\right) .
$$

Combining (3.5),(3.16), (3.18), and (3.23), we have shown that

$$
E_{n, \beta} e^{x \lambda_{k}^{\prime}}=\exp \left(\frac{u \mu_{n, k}}{\sigma_{n}}+\frac{u^{2} \kappa_{\beta}^{2}(0)}{2}\right)(1+o(1)) .
$$

Therefore, (3.4), and so (3.2), hold true as desired.

The proof of (3.3) is completely similar; we only remark that the difference mainly stems from (3.10). The limit variance $\kappa_{\beta}^{2}(0)$ in the case $\beta>1$ (see (3.2)) consists of two parts: one is given by the second term of the right hand side of (3.10), while the other is induced by the third term of the right hand side of (3.15). When $\beta=1$,

$$
\sigma_{n, k}^{2}=\sum_{j=k}^{\infty} \frac{j e^{-j h_{n}}}{\left(1-e^{-j h_{n}}\right)^{2}}=\sigma_{n}^{2}\left|\ln h_{n}\right|(1+o(1)) .
$$

In this case we choose $\sigma_{n} \sqrt{\left|\ln h_{n}\right|}$ as a normalizing constant. Given $u \in \mathbf{R}$, let $x=u /\left(\sigma_{n} \sqrt{\left|\ln h_{n}\right|}\right)$, then (3.10) becomes

$$
\sum_{j=k}^{\infty} j \ln \frac{1-e^{-j \tau^{*}}}{1-e^{x} e^{-j \tau^{*}}}=\frac{\mu_{n, k} u}{\sigma_{n} \sqrt{\left|\ln h_{n}\right|}}+\frac{u^{2}}{2}+o(1) .
$$

The third term of the right-hand side of (3.15) is now negligible. The theorem is proved.

Theorem 3.1 corresponds to the end of partitions. We consider the fluctuations in the deep bulk of partitions below. Let

$$
V_{n}(t)=\frac{1}{\sigma_{n}}\left(\lambda_{\left[t / h_{n}\right]}^{\prime}-\mu_{n,\left[t / h_{n}\right]}\right), \quad t \geqslant 0,
$$

where $\lambda_{0}^{\prime}=\lambda_{1}^{\prime}$. 
Theorem 3.2. Under $P_{n, \beta}$ with $\beta>-1$, we have as $n \rightarrow \infty$

(1) for each $t>0$,

$$
V_{n}(t) \stackrel{d}{\longrightarrow} V(t)
$$

where $V(t)$ is a normal random variable with zero mean and variance

$$
\kappa_{\beta}^{2}(t)=\sigma_{\beta}^{2}(t)-\frac{1}{\Gamma(\beta+3) \zeta(\beta+2)}\left(\sigma_{\beta+1}^{2}(t)\right)^{2} .
$$

(2) for $0<t_{1}<t_{2}<\cdots<t_{m}<\infty$,

$$
\left(V_{n}\left(t_{1}\right), V_{n}\left(t_{2}\right), \ldots, V_{n}\left(t_{m}\right)\right) \stackrel{d}{\longrightarrow}\left(V\left(t_{1}\right), V\left(t_{2}\right), \ldots, V\left(t_{m}\right)\right),
$$

where $\left(V\left(t_{1}\right), V\left(t_{2}\right), \ldots, V\left(t_{m}\right)\right)$ is a Gaussian vector with covariance structure

$$
\operatorname{Cov}(V(s), V(t))=\sigma_{\beta}^{2}(t)-\frac{\sigma_{\beta+1}^{2}(s) \sigma_{\beta+1}^{2}(t)}{\Gamma(\beta+3) \zeta(\beta+2)}, \quad s<t .
$$

(3) Each separable version of $V$ is continuous in $(0, \infty)$.

The proof is similar to that of Theorem 3.1 and is left to the interest reader. The hypothesis $\beta>1$ was used to guarantee the integral $\int_{0}^{\infty}\left(u^{\beta} e^{-u} /\left(1-e^{-u}\right)^{2}\right) d u<\infty$. However, for each fixed $t>0$ we can weaken this requirement.

Having convergence of finite-dimensional distributions, it is natural to expect the weak convergence of $V_{n}(\cdot)$ in function space. This requires that $V_{n}(\cdot)$ satisfies a certain uniform tightness, namely for every $\varepsilon>0$

$$
\lim _{\delta \rightarrow 0} \lim _{n \rightarrow \infty} P_{n, \beta}\left(\sup _{|t-s| \leqslant \delta}\left|V_{n}(t)-V_{n}(s)\right| \geqslant \varepsilon\right)=0 .
$$

Note a similar uniform tightness was proved true in the independent case (see Theorem 2.3). Unfortunately, we can only prove a weaker version of (3.24), i.e., stochastic equi-continuity holds for $V_{n}(\cdot)$. However, this will still guarantee convergence in distribution of every integral functional from a broad class; see [12, section 7, Chap. IX].

Let $\mathscr{G}$ be the set of all continuous functions $g(t, x)$ in $\mathbf{R}_{+} \times \mathbf{R}$ such that for some $\omega$ and $\nu$ with $(\beta+1) /(6(\beta+2)) \nu>\omega$ if $\beta>2$ and $(\beta-1) /(2(\beta+2)) \nu>\omega$ if $1<\beta \leqslant 2$

$$
|g(t, x)|=O\left(e^{\omega t}|x|^{\nu}\right)
$$

uniformly over $\mathbf{R}_{+} \times \mathbf{R}$.

Theorem 3.3. Under $P_{n, \beta}$ with $\beta>1$, we have for each $g \in \mathscr{G}$

$$
\int_{0}^{\infty} g\left(t, V_{n}(t)\right) d t \stackrel{d}{\longrightarrow} \int_{0}^{\infty} g(t, V(t)) d t
$$


We shall apply the Gikhman-Skorokhod method to prove (3.25). To this end, we need the following lemmas to show that $V_{n}(\cdot)$ satisfies the stochastic equi-continuity condition.

Lemma 3.2. Let $1 \leqslant k_{1}<k_{2}$ and

$$
\eta_{n}= \begin{cases}h_{n}^{(\beta+1) / 3}, & \beta>2 \\ h_{n} \ln ^{-1 / 3} n, & \beta=2 \\ h_{n}, & 1<\beta<2 .\end{cases}
$$

Then for each $\eta$ such that

$$
\eta= \begin{cases}O\left(\eta_{n}\right), & \beta \geqslant 2 \\ o\left(\eta_{n}\right), & 1<\beta<2\end{cases}
$$

we have

$$
E_{n, \beta} \exp \left(\eta\left(\lambda_{k_{1}}^{\prime}-\lambda_{k_{2}}^{\prime}-\left(\mu_{n, k_{1}}-\mu_{n, k_{2}}\right)\right)\right) \leqslant \exp \left(\frac{\eta^{2}}{2}\left(\sigma_{n, k_{1}}^{2}-\sigma_{n, k_{2}}^{2}\right)\right) .
$$

P r o o f. We can adapt a completely similar argument to the proposition of [20] with some modifications given in the proof of Theorem 3.1. The lemma is proved.

Lemma 3.3. Assume $\beta>1$. Then for each $\varepsilon>0$

$$
\lim _{\delta \rightarrow 0} \lim _{n \rightarrow \infty} \sup _{|t-s| \leqslant \delta} P_{n, \beta}\left(\left|V_{n}(t)-V_{n}(s)\right| \geqslant \varepsilon\right)=0
$$

and for $m \geqslant 1$

$$
E_{n, \beta}\left|V_{n}(t)\right|^{m}=O\left(\sigma_{\beta}^{m}(t)+\left(\sigma_{n} \eta_{n}\right)^{-m}(\ln n)^{m}\right) .
$$

P r o o f. Assume $0 \leqslant t<s$. By the Euler-Maclaurin formula, we have

$$
\sigma_{n ; t, s}^{2}=: \sigma_{n,\left[t / h_{n}\right]}^{2}-\sigma_{n,\left[s / h_{n}\right]}^{2}=\frac{1}{h_{n}^{\beta+1}} \int_{t}^{s} \frac{u^{\beta} e^{-u}}{\left(1-e^{-u}\right)^{2}} d u+O\left(\frac{1}{h_{n}^{\beta}}\right),
$$

and so

$$
\frac{\sigma_{n ; t, s}^{2}}{\sigma_{n}^{2}} \longrightarrow \int_{t}^{s} \frac{u^{\beta} e^{-u}}{\left(1-e^{-u}\right)^{2}} d u=\sigma_{\beta}^{2}(t)-\sigma_{\beta}^{2}(s) .
$$

By Lemma 3.2, we obtain for each $\eta$ satisfying condition (3.26)

$$
\begin{aligned}
E_{n, \beta} e^{\sigma_{n} \eta\left(V_{n}(t)-V_{n}(s)\right)} & \leqslant \exp \left(\frac{\eta^{2}}{2} \sigma_{n ; t, s}^{2}\right) \\
& \leqslant a \exp \left(\frac{\sigma_{n}^{2} \eta^{2}}{2}\left(\sigma_{\beta}^{2}(t)-\sigma_{\beta}^{2}(s)\right)\right),
\end{aligned}
$$

where $a>0$ is a numerical constant. Consequently, given $x>0$,

$$
P_{n, \beta}\left(\left|V_{n}(t)-V_{n}(s)\right| \geqslant x\right) \leqslant a \exp \left(\frac{\sigma_{n}^{2} \eta^{2}}{2}\left(\sigma_{\beta}^{2}(t)-\sigma_{\beta}^{2}(s)\right)-\sigma_{n} \eta x\right) .
$$


Note that the quadratic function in $\eta$ in the above exponent attains its minimum at

$$
\eta_{n}(x)=: \frac{x}{\sigma_{n}\left(\sigma_{\beta}^{2}(t)-\sigma_{\beta}^{2}(s)\right)} .
$$

Put $x_{0}=\sigma_{n} \eta\left(\sigma_{\beta}^{2}(t)-\sigma_{\beta}^{2}(s)\right)$. Below we refine the upper bound (3.29).

(i) $x \leqslant x_{0}$. Plugging $\eta=\eta_{n}(x)$ into (3.29) yields

$$
P_{n, \beta}\left(\left|V_{n}(t)-V_{n}(s)\right| \geqslant x\right) \leqslant a \exp \left(-\frac{x^{2}}{2\left(\sigma_{\beta}^{2}(t)-\sigma_{\beta}^{2}(s)\right)}\right) .
$$

(ii) $x>x_{0}$. Plugging $\eta=\eta_{n}\left(x_{0}\right)$ into (3.29) yields

$$
P_{n, \beta}\left(\left|V_{n}(t)-V_{n}(s)\right| \geqslant x\right) \leqslant a \exp \left(-\frac{\sigma_{n} \eta x}{2}\right) .
$$

Now choose $\eta=\eta_{n}$ if $\beta \geqslant 2$; otherwise $\eta=\eta_{n}(\ln n)^{-1}$, where $\eta_{n}$ is given by Lemma 3.2. Then for every $\varepsilon>0$ we have

$$
P_{n, \beta}\left(\left|V_{n}(t)-V_{n}(s)\right| \geqslant \varepsilon\right) \leqslant a \exp \left(-\frac{\varepsilon^{2}}{2\left(\sigma_{\beta}^{2}(t)-\sigma_{\beta}^{2}(s)\right)}\right)+a \exp \left(-\frac{\sigma_{n} \eta_{n} \varepsilon}{2 \ln n}\right) .
$$

Note $\left(\sigma_{n} \eta_{n}\right) /(2 \ln n) \rightarrow \infty$ for any $\beta>1$. Letting $n \rightarrow \infty$ and then letting $\delta \rightarrow 0$ gives $(3.27)$.

Further we turn to prove (3.28). Analogously to (3.30) and (3.31), it follows that

$$
P_{n, \beta}\left(\left|V_{n}(t)\right| \geqslant x\right) \leqslant \begin{cases}a \exp \left(-\frac{x^{2}}{2 \sigma_{\beta}^{2}(t)}\right), & x \leqslant x_{0}, \\ a \exp \left(-\frac{\sigma_{n} \eta x}{2}\right), & x>x_{0} .\end{cases}
$$

where $x_{0}=\sigma_{n} \eta \sigma_{\beta}^{2}(t)$.

Choosing $\eta$ as above and integrating by part we obtain

$$
E_{n, \beta}\left|V_{n}(t)\right|^{m}=O\left(\sigma_{\beta}^{m}(t)+\left(\sigma_{n} \eta_{n}\right)^{-m}(\ln n)^{m}\right) .
$$

The proof is complete.

Proof of $\mathrm{The}$ orem 3.3. Fix $g \in \mathscr{G}$. First, it follows from (3.27) and the Gikhman-Skorokhod lemma (see [12, Theorem 2, section 7, Chap. IX]) that for any $M>0$

$$
\int_{0}^{M} g\left(t, V_{n}(t)\right) d t \stackrel{d}{\longrightarrow} \int_{0}^{M} g(t, V(t)) d t
$$

Also, we have as $M \rightarrow \infty$

$$
\int_{M}^{\infty}|g(t, V(t))| d t \stackrel{P}{\longrightarrow} 0
$$


Indeed, for $g \in \mathscr{G}$

$$
\int_{M}^{\infty} E|g(t, V(t))| d t \leqslant \int_{M}^{\infty} e^{\omega t} E|V(t)|^{\nu} d t
$$

Since $V(t)$ is normal with zero mean and variance $\operatorname{Var}(V(t))=\kappa_{\beta}^{2}(t)$, then

$$
E|V(t)|^{\nu} \leqslant A_{\nu} \kappa_{\beta}^{\nu}(t) \leqslant A_{\nu} \sigma_{\beta}^{\nu}(t)
$$

where $A_{\nu}$ is a constant depending on $\nu$.

In addition, obviously $\nu / 2>\omega$ by the hypothesis on $\nu$ and $\omega$. This gives

$$
\begin{aligned}
\int_{M}^{\infty} e^{\omega t} \sigma_{\beta}^{\nu}(t) d t & =\int_{M}^{\infty} e^{\omega t}\left(\int_{t}^{\infty} \frac{u^{\beta} e^{-u}}{\left(1-e^{-u}\right)^{2}} d u\right)^{\nu / 2} d t \\
& =O\left(M^{\beta \nu / 2} e^{-(\nu / 2-\omega) M}\right) \longrightarrow 0 \quad(M \rightarrow \infty)
\end{aligned}
$$

Thus (3.33) goes to 0 as $M \rightarrow \infty$, as desired.

Further we prove as $n \rightarrow \infty, M \rightarrow \infty$

$$
\int_{M}^{\infty} g\left(t, V_{n}(t)\right) d t \stackrel{P}{\longrightarrow} 0
$$

As in (3.33), we have

$$
\int_{M}^{\ln n} E_{n, \beta}\left|g\left(t, V_{n}(t)\right)\right| d t \leqslant \int_{M}^{\ln n} e^{\omega t} E_{n, \beta}\left|V_{n}(t)\right|^{\nu} d t .
$$

By virtue of (3.28), the right-hand side of (3.35) is bounded up to a factor by

$$
\int_{M}^{\infty} e^{\omega t} \sigma_{\beta}^{\nu}(t) d t+\left(\sigma_{n} \eta_{n}\right)^{-\nu}(\ln n)^{\nu} n^{\omega}
$$

which in turn goes to 0 as $n \rightarrow \infty, M \rightarrow \infty$ by the definition of $\eta_{n}$ and the hypotheses on $\nu$ and $\omega$.

Turn to the integral $\int_{\ln n}^{\infty} g\left(t, V_{n}(t)\right) d t$. It follows from (1.4), that

$$
\lim _{n \rightarrow \infty} P_{n, \beta}\left(\lambda_{1}>\left(\frac{\beta+1}{\beta+2}+\varepsilon\right) \frac{\ln n}{h_{n}}\right)=0 \quad \forall \varepsilon>0 .
$$

In particular, the event $\left\{\lambda_{1} \leqslant \ln n / h_{n}\right\}$ occurs with a high probability. On the other hand, on this event $\lambda_{\left[t / h_{n}\right]}^{\prime}=0$ whenever $t>\ln n$. Thus we have

$$
V_{n}(t)=-\frac{\mu_{n,\left[t / h_{n}\right]}}{\sigma_{n}}
$$


and so

$$
\begin{aligned}
\int_{\ln n}^{\infty}\left|g\left(t, V_{n}(t)\right)\right| d t & \leqslant \int_{\ln n}^{\infty} e^{\omega t}\left|V_{n}(t)\right|^{\nu} d t \\
& \leqslant \frac{1}{\sigma_{n}^{\nu}} \int_{\ln n}^{\infty} e^{\omega t}\left(\sum_{k=\left[t / h_{n}\right]}^{\infty} \frac{k^{\beta} e^{-k h_{n}}}{1-e^{-k h_{n}}}\right)^{\nu} d t \\
& \rightarrow 0 \quad(n \rightarrow \infty) .
\end{aligned}
$$

Combined, we have shown (3.34). The proof of Theorem 3.3 is now complete.

4. Normal convergence of $d_{\lambda}$. In this section we are concerned with the number $d_{\lambda}$ of the standard Young tableaux of a Young diagram $\lambda \in \mathscr{P}_{n}$. Let us start with two useful expressions for the computation of $d_{\lambda}$.

Assume $\lambda=\left(\lambda_{1}, \ldots, \lambda_{l}\right)$ and $\lambda^{\prime}=\left(\lambda_{1}^{\prime}, \ldots, \lambda_{\lambda_{1}}^{\prime}\right)$. A so-called hook formula discovered by Frames, Robinson, and Thrall [9] reads

$$
d_{\lambda}=\frac{n !}{\prod_{(i, j) \in \lambda}\left(\lambda_{i}-j+\lambda_{j}^{\prime}-i+1\right)},
$$

where the product is over all $n$ unit cells in the diagram $\lambda$. Trivially, $d_{\lambda}=d_{\lambda^{\prime}}$.

An alternative expression, due to Frobenius [11], is given by

$$
d_{\lambda}=\frac{n !}{H_{\lambda}}
$$

where

$$
H_{\lambda}=\frac{\prod_{i=1}^{l}\left(\lambda_{i}+l-i\right) !}{\prod_{1 \leqslant i<j \leqslant l}\left(\lambda_{i}-i-\left(\lambda_{j}-j\right)\right)} .
$$

Considerably deeper is the RSK correspondence between the set of partitions of the integer $n$ and the symmetric group $S_{n}$. The RSK correspondence actually gives a procedure for obtaining Young tableaux with the help of permutations, from which the Burnside identity

$$
\sum_{\lambda \in \mathscr{P}_{n}} \frac{d_{\lambda}^{2}}{n !}=1
$$

follows.

In the remarkable series of papers [22] and [20], [21] studied at length the likely magnitude of $d_{\lambda}$ under $\left(\mathscr{P}_{n}, P_{n, 0}\right)$. In particular, $\ln \left(d_{\lambda}^{2} / n\right.$ !) is asymptotically normal with mean $-A n$ ( $A$ is a positive constant) and standard deviation of order $n^{3 / 4}$.

The aim of this section is to find the limiting distribution of $\ln \left(d_{\lambda}^{\beta+2} / n\right.$ !) under $\left(\mathscr{P}_{n}, P_{n, \beta}\right), \beta>0$. Our main result is the following theorem. 
Theorem 4.1. Under $\left(\mathscr{P}_{n}, P_{n, \beta}\right)$ with $\beta>1$, we have as $n \rightarrow \infty$

$$
h_{n}^{(\beta+3) / 2}\left(\ln \frac{d_{\lambda}}{(n !)^{1 /(\beta+2)}}-b_{n}\right) \stackrel{d}{\longrightarrow} N\left(0, \Lambda^{2}\right),
$$

where $q=e^{-h_{n}}$,

$$
\begin{aligned}
b_{n}= & \frac{\beta+1}{\beta+2} \sum_{k=1}^{\infty} \mu_{n, k} \ln n-\sum_{k=1}^{\infty} \mu_{n, k} \ln \mu_{n, k}+\sum_{k=1}^{\infty} \mu_{n, k}-\frac{\beta+1}{\beta+2} n \\
& -\frac{\beta+1}{\beta+2}(\ln \Gamma(\beta+2) \zeta(\beta+2))\left(\sum_{k=1}^{\infty} \mu_{n, k}-n\right)
\end{aligned}
$$

and

$$
\Lambda^{2}=\int_{0}^{\infty} \int_{0}^{\infty} \operatorname{Cov}(V(x), V(y)) \ln \Psi_{\beta}(x) \ln \Psi_{\beta}(y) d x d y .
$$

$\mathrm{R}$ e $\mathrm{m}$ a r k 4.1. We conjecture that (4.3) is also valid for $\beta \in(0,1)$. However, the present technique does not allow us to do it. The centering constant $b_{n}$ in (4.4) is of the order $n$, which can be seen from the EulerMaclaurin formula.

In order to prove Theorem 4.1, we need the following lemma.

Lemma 4.1. Assume $\beta>1$. Then

$$
\begin{gathered}
\max _{n, k \geqslant 1} \frac{\sigma_{n, k}^{2}}{\mu_{n, k}} \leqslant C_{\beta}, \\
\lim _{n \rightarrow \infty} P_{n, \beta}\left(\sum_{k=1}^{\lambda_{1}} \frac{\sigma_{n, k}}{\mu_{n, k}} \geqslant \frac{C_{\beta}}{h_{n}^{(\beta-1) / 2}}\right)=0, \\
\lim _{n \rightarrow \infty} P_{n, \beta}\left(\max _{1 \leqslant k \leqslant \lambda_{1}} \frac{\left|\lambda_{k}^{\prime}-\mu_{n, k}\right|}{\sigma_{n, k}} \geqslant \sqrt{3 \ln n}\right)=0,
\end{gathered}
$$

where $\mu_{n, k}$ and $\sigma_{n, k}^{2}$ are as in (3.1).

$\mathrm{P} r$ o o f. We start with the proof of (4.5). Recall the definition of $f$ in (2.22). Then by the Euler-Maclaurin sum formula, we have for $k \geqslant 1$

$$
\mu_{n, k}=\frac{1}{h_{n}^{\beta+1}} \int_{k h_{n}}^{\infty} f(x) d x+\frac{1}{2 h_{n}^{\beta}} f\left(k h_{n}\right)+R_{n, k}(f, 1),
$$

where $R_{n, k}(f, 1)$ is an error term satisfying

$$
\left|R_{n, k}(f, 1)\right| \leqslant \frac{1}{12 h_{n}^{\beta-1}}\left(\left|f^{\prime}\left(k h_{n}\right)\right|+\int_{k h_{n}}^{\infty}\left|f^{\prime \prime}(x)\right| d x\right) .
$$

Similarly, putting

$$
f_{2}(x)=\frac{x^{\beta} e^{-x}}{\left(1-e^{-x}\right)^{2}}
$$


we have for $k \geqslant 1$

$$
\sigma_{n, k}^{2}=\frac{1}{h_{n}^{\beta+1}} \int_{k h_{n}}^{\infty} f_{2}(x) d x+\frac{1}{2 h_{n}^{\beta}} f_{2}\left(k h_{n}\right)+R_{n, k}\left(f_{2}, 1\right),
$$

where $R_{n, k}\left(f_{2}, 1\right)$ is an error term satisfying

$$
\left|R_{n, k}\left(f_{2}, 1\right)\right| \leqslant \frac{1}{12 h_{n}^{\beta-1}}\left(\left|f_{2}^{\prime}\left(k h_{n}\right)\right|+\int_{k h_{n}}^{\infty}\left|f_{2}^{\prime \prime}(x)\right| d x\right) .
$$

A key observation is

$$
\frac{c_{\beta}}{h_{n}^{\beta+1}} \int_{k h_{n}}^{\infty} f(x) d x \leqslant \mu_{n, k} \leqslant \frac{C_{\beta}}{h_{n}^{\beta+1}} \int_{k h_{n}}^{\infty} f(x) d x
$$

and

$$
\sigma_{n, k}^{2} \leqslant \frac{C_{\beta}}{h_{n}^{\beta+1}} \int_{k h_{n}}^{\infty} f_{2}(x) d x .
$$

We only show (4.10), the other is very similar. Fix $M>0$ such that $e^{M}>2$. We consider two subsets of integers separately: $\left\{k: k h_{n} \leqslant M\right\}$ and $\left\{k: k h_{n}>\right.$ $M$ \}. First, note that

$$
C=: \sup _{0 \leqslant x<\infty} \frac{x e^{-x}}{1-e^{-x}}<\infty, \quad \int_{0}^{\infty} f(x) d x<\infty .
$$

Also,

$$
f^{\prime}(x)=\frac{\beta x^{\beta-1} e^{-x}}{1-e^{-x}}-\frac{x^{\beta} e^{-x}}{\left(1-e^{-x}\right)^{2}}
$$

and

$$
\begin{aligned}
f^{\prime \prime}(x)= & \beta(\beta-1) \frac{x^{\beta-2} e^{-x}}{1-e^{-x}}-\frac{\beta x^{\beta-1} e^{-x}}{1-e^{-x}} \\
& -\frac{\beta x^{\beta-1} e^{-x}}{\left(1-e^{-x}\right)^{2}}+\frac{x^{\beta} e^{-x}}{\left(1-e^{-x}\right)^{2}}+2 \frac{x^{\beta} e^{-x}}{\left(1-e^{-x}\right)^{3}} .
\end{aligned}
$$

(i) Assume $k$ is such that $k h_{n} \leqslant M$. Then we have the following relations:

$$
\begin{gathered}
\int_{M}^{\infty} f(x) d x \leqslant \int_{k h_{n}}^{\infty} f(x) d x \leqslant \int_{0}^{\infty} f(x) d x, \\
f\left(k h_{n}\right) \leqslant C M^{\beta-1},, \\
\left|f^{\prime}\left(k h_{n}\right)\right| \leqslant C_{\beta}\left(k h_{n}\right)^{\beta-2} \leqslant \begin{cases}C_{\beta} M^{\beta-2}, & \beta>2, \\
C_{\beta} h_{n}^{\beta-2}, & 1<\beta \leqslant 2,\end{cases}
\end{gathered}
$$

and

$$
\int_{k h_{n}}^{\infty}\left|f^{\prime \prime}(x)\right| d x \leqslant \frac{C_{\beta}}{h_{n}}
$$


(ii) Assume $k$ is such that $k h_{n}>M$. Then we have the following relations:

$$
\begin{gathered}
c_{\beta}\left(k h_{n}\right)^{\beta} e^{-k h_{n}} \leqslant \int_{k h_{n}}^{\infty} f(x) d x \leqslant C_{\beta}\left(k h_{n}\right)^{\beta} e^{-k h_{n}}, \\
f\left(k h_{n}\right) \leqslant 2\left(k h_{n}\right)^{\beta} e^{-k h_{n}}, \quad f^{\prime}\left(k h_{n}\right) \leqslant C_{\beta}\left(k h_{n}\right)^{\beta} e^{-k h_{n}}, \\
\int_{k h_{n}}^{\infty} f^{\prime \prime}(x) d x \leqslant C_{\beta}\left(k h_{n}\right)^{\beta} e^{-k h_{n}} .
\end{gathered}
$$

Combining these with (4.8) easily yields (4.10).

In view of (4.10) and (4.11), it follows that

$$
\max _{n, k \geqslant 1} \frac{\sigma_{n, k}^{2}}{\mu_{n, k}} \leqslant \frac{C_{\beta}}{c_{\beta}} \cdot \max _{n, k \geqslant 1} \frac{\int_{k h_{n}}^{\infty} f_{2}(x) d x}{\int_{k h_{n}}^{\infty} f(x) d x},
$$

which implies (4.5).

Let us turn to the sum estimation in (4.6). Fix $\varepsilon>0$. Let

$$
B_{n}=\frac{1}{h_{n}}\left(\frac{\beta+1}{\beta+2} \ln n+(\beta+\varepsilon) \ln \ln n\right) .
$$

Then by (1.4), it follows that

$$
\lim _{n \rightarrow \infty} P_{n, \beta}\left(\lambda_{1}>B_{n}\right)=0 .
$$

So it suffices to prove

$$
\sum_{1 \leqslant k \leqslant B_{n}} \frac{\sigma_{n, k}}{\mu_{n, k}} \leqslant \frac{C_{\beta}}{h_{n}^{(\beta-1) / 2}} .
$$

Again, according to (4.10) and (4.11), it follows that

$$
\sum_{1 \leqslant k \leqslant B_{n}} \frac{\sigma_{n, k}}{\mu_{n, k}} \leqslant C_{\beta} h_{n}^{(\beta+1) / 2} \sum_{1 \leqslant k \leqslant B_{n}} \frac{\left(\int_{k h_{n}}^{\infty} f_{2}(x) d x\right)^{1 / 2}}{\int_{k h_{n}}^{\infty} f(x) d x} .
$$

As in the proof of (4.10) above, split the sum of (4.16) into two subsums over $\left\{k: k h_{n} \leqslant M\right\}$ and $\left\{k: k h_{n}>M\right\}$. It is easy to see that

$$
\sum_{k: k h_{n} \leqslant M} \frac{\left(\int_{k h_{n}}^{\infty} f_{2}(x) d x\right)^{1 / 2}}{\int_{k h_{n}}^{\infty} f(x) d x} \leqslant \frac{C_{\beta} M}{h_{n}}
$$

and

$$
\sum_{M / h_{n} \leqslant k \leqslant B_{n}} \frac{\left(\int_{k h_{n}}^{\infty} f_{2}(x) d x\right)^{1 / 2}}{\int_{k h_{n}}^{\infty} f(x) d x} \leqslant C_{\beta}(\ln n)^{\varepsilon / 2} .
$$

These together with (4.16) imply the proof of (4.6). 
Finally, we shall prove (4.7). By (4.14), it suffices to prove

$$
P_{n, \beta}\left(\max _{1 \leqslant k \leqslant B_{n}} \frac{\left|\lambda_{k}^{\prime}-\mu_{n, k}\right|}{\sigma_{n, k}} \geqslant \sqrt{3 \ln n}\right) \longrightarrow 0 .
$$

To this end, let us first prove for each $b>0$,

$$
P_{\beta, q}\left(\frac{\left|\lambda_{k}^{\prime}-\mu_{n, k}\right|}{\sigma_{n, k}} \geqslant \sqrt{b \ln n}\right) \leqslant 2 \exp \left(-\frac{b}{3} \ln n\right), \quad k \geqslant 1,
$$

where $q=e^{-h_{n}}$. As in Theorem 2.1, we have for every $x \in \mathbf{R}$

$$
E_{\beta, q} e^{x \lambda_{k}^{\prime}}=\exp \left(x \mu_{n, k}+\frac{x^{2}}{2} \sigma_{n, k}^{2}+O\left(|x|^{3} \sum_{j=k}^{\infty} \frac{j^{\beta} e^{-3 j h_{n}}}{\left(1-e^{-j h_{n}}\right)^{3}}\right)\right) .
$$

In particular, set $x=\sqrt{b \ln n} / \sigma_{n, k}$. We only need to check

$$
\frac{(\ln n)^{3 / 2}}{\sigma_{n, k}^{3}} \sum_{j=k}^{\infty} \frac{j^{\beta} e^{-3 j h_{n}}}{\left(1-e^{-j h_{n}}\right)^{3}}=o(1)
$$

uniformly in $k \geqslant 1$. In turn, this can be proved in a similar way to (4.5).

Estimate (4.18) now easily follows from Chebyschev's inequality. To prove (4.17), observe the following relation: for any set $B$,

$$
P_{n, \beta}\left\{\left(r_{1}, \ldots, r_{n}\right) \in B\right\} \leqslant \frac{1}{P_{\beta, q}(|\lambda|=n)} P_{\beta, q}\left\{\left(r_{1}, \ldots, r_{n}\right) \in B\right\} .
$$

Besides, a similar argument to Lemma 4.2 [10] shows

$$
P_{\beta, q}(|\lambda|=n)=\frac{h_{n}^{(\beta+3) / 2}}{\sqrt{2 \pi \Gamma(\beta+3) \zeta(\beta+3,0)}}(1+o(1)) .
$$

These together with (4.18) immediately imply (4.7).

Pr o of of $\mathrm{Th}$ e or e m 4.1. Since $d_{\lambda}=d_{\lambda^{\prime}}$, it is sufficient for us to prove

$$
h_{n}^{(\beta+3) / 2}\left(\ln \frac{d_{\lambda^{\prime}}}{(n !)^{1 /(\beta+2)}}-b_{n}\right) \stackrel{d}{\longrightarrow} N\left(0, \Lambda^{2}\right),
$$

According to the Frobenius formula (4.2),

$$
\ln \frac{d_{\lambda^{\prime}}}{(n !)^{1 /(\beta+2)}}=\ln \frac{1}{H_{\lambda^{\prime}}}+\frac{\beta+1}{\beta+2} \ln n !
$$

For any partition $\lambda$ and its dual $\lambda^{\prime}$, it follows that

$$
\frac{1}{H_{\lambda^{\prime}}}=\frac{\prod_{1 \leqslant i<j \leqslant \lambda_{1}}\left(\lambda_{i}^{\prime}-\lambda_{j}^{\prime}+j-i\right)}{\prod_{1 \leqslant i \leqslant \lambda_{1}}\left(\lambda_{i}^{\prime}-i+\lambda_{1}\right) !}=: \frac{M_{n}}{N_{n}},
$$


and so

$$
\ln \frac{1}{H_{\lambda^{\prime}}}=\ln M_{n}-\ln N_{n}
$$

Use (4.14),

$$
\ln M_{n}=\sum_{1 \leqslant i<j \leqslant \lambda_{1}} \ln \left(\lambda_{i}^{\prime}-\lambda_{j}^{\prime}+j-i\right) \leqslant \lambda_{1}^{2} \ln n=O_{p}\left(\frac{\ln ^{3} n}{h_{n}^{2}}\right),
$$

where and in the sequel the notation $X_{n}=Y_{n}+O_{p}\left(c_{n}\right)$ means $P\left(\left|X_{n}-Y_{n}\right| \geqslant\right.$ $\left.M c_{n}\right) \rightarrow 0$ for some $M>0$.

Note $\ln M_{n}$ in (4.21) is negligible compared to the variance $h_{n}^{-(\beta+3) / 2}$ when $\beta>1$. The normal fluctuation in (4.20) mainly comes from the term $\ln N_{n}$. By Stirling's approximation for factorial and the upper bound for $\lambda_{1}$, we easily see, that

$$
\begin{aligned}
\ln N_{n} & =\sum_{1 \leqslant i \leqslant \lambda_{1}} \ln \left(\lambda_{i}^{\prime}-i+\lambda_{1}\right) ! \\
& =\sum_{1 \leqslant i \leqslant \lambda_{1}} \phi\left(\lambda_{i}^{\prime}-i+\lambda_{1}\right)+O_{p}\left(\frac{\ln ^{2} n}{h_{n}}\right),
\end{aligned}
$$

where $\phi(x)=x \ln x-x$.

On the other hand, the Taylor expansion for the function $\phi$ : is for any $x_{0}$

$$
\phi(x)=\phi\left(x_{0}\right)+\phi^{\prime}\left(x_{0}\right)\left(x-x_{0}\right)+\int_{x_{0}}^{x} \phi^{\prime \prime}(t)(x-t) d t .
$$

We get

$$
\begin{aligned}
\sum_{1 \leqslant i \leqslant \lambda_{1}} \phi\left(\lambda_{i}^{\prime}-i+\lambda_{1}\right)= & \sum_{1 \leqslant i \leqslant \lambda_{1}} \phi\left(\mu_{n, i}-i+\lambda_{1}\right) \\
& +\sum_{1 \leqslant i \leqslant \lambda_{1}}\left(\lambda_{i}^{\prime}-\mu_{n, i}\right) \ln \left(\mu_{n, i}-i+\lambda_{1}\right) \\
& +\sum_{1 \leqslant i \leqslant \lambda_{1}} R_{\phi, i},
\end{aligned}
$$

where the error term satisfies

$$
\left|R_{\phi, i}\right| \leqslant \frac{\left(\lambda_{i}^{\prime}-\mu_{n, i}\right)^{2}}{\mu_{n, i}-i+\lambda_{1}} .
$$

Now we can use Lemma 4.1 to show that the square error term is negligible:

$$
\sum_{1 \leqslant i \leqslant \lambda_{1}} \frac{\left(\lambda_{i}^{\prime}-\mu_{n, i}\right)^{2}}{\mu_{n, i}-i+\lambda_{1}}=O_{p}\left(\ln n \sum_{1 \leqslant i \leqslant \lambda_{1}} \frac{\sigma_{i}^{2}}{\mu_{n, i}}\right)=O_{p}\left(\frac{\ln ^{2} n}{h_{n}}\right) .
$$


The second term of the right-hand side of (4.23) gives the linear approximation of $\ln N_{n}$. We proceed to simplify the logarithmic factor. Let

$$
\nu_{n, i}=\frac{1}{h_{n}^{\beta+1}} \Psi_{\beta}\left(i h_{n}\right)
$$

where $\Psi_{\beta}$ is as in Lemma 1.2 , then it is easy to see, that

$$
\begin{aligned}
& \sum_{1 \leqslant i \leqslant \lambda_{1}}\left(\lambda_{i}^{\prime}-\mu_{n, i}\right) \ln \left(\mu_{n, i}-i+\lambda_{1}\right) \\
& =\sum_{1 \leqslant i \leqslant \lambda_{1}}\left(\lambda_{i}^{\prime}-\mu_{n, i}\right) \ln \nu_{n, i}+\sum_{1 \leqslant i \leqslant \lambda_{1}} R_{\ln , i},
\end{aligned}
$$

where the error term satisfies

$$
\left|R_{\ln , i}\right| \leqslant \frac{\left|\lambda_{i}^{\prime}-\mu_{n, i}\right|}{\nu_{n, i} \vee \mu_{n, i}} \cdot\left|\mu_{n, i}-\nu_{n, i}+\lambda_{1}-i\right| .
$$

Once again, the error term in (4.24) is negligible. Indeed, by Lemma 4.1 it suffices to control

$$
\sum_{1 \leqslant i \leqslant \lambda_{1}} \frac{\sigma_{n, i}}{\nu_{n, i} \vee \mu_{n, i}}\left|\mu_{n, i}-\nu_{n, i}\right|=O_{p}\left(\frac{1}{h_{n}^{(\beta+1) / 2}}\right) .
$$

This in turn can be proved as in the proof of Lemma 4.1.

Further, let us turn to the first term of the right-hand side of (4.24) and (4.23), respectively. It obviously follows that

$$
\sum_{1 \leqslant i \leqslant \lambda_{1}}\left(\lambda_{i}^{\prime}-\mu_{n, i}\right)=\sum_{1 \leqslant i \leqslant \lambda_{1}} \lambda_{i}^{\prime}-\sum_{1 \leqslant i \leqslant \lambda_{1}} \mu_{n, i}=n-\sum_{i=1}^{\infty} \mu_{n, i}+\sum_{i>\lambda_{1}} \mu_{n, i}
$$

and

$$
\begin{aligned}
\sum_{i>\lambda_{1}} \mu_{n, i} & =\sum_{i>\lambda_{1}} \sum_{k \geqslant i} \frac{k^{\beta} e^{-k h_{n}}}{1-e^{-k h_{n}}}=\sum_{k>\lambda_{1}} \frac{k^{\beta+1} e^{-k h_{n}}}{1-e^{-k h_{n}}} \\
& \leqslant \frac{1}{h_{n}^{\beta+2}} \int_{\lambda_{1} h_{n}}^{\infty} \frac{u^{\beta+1} e^{-u}}{1-e^{-u}} d u=O_{p}\left(\frac{1}{h_{n}}(\ln n)^{\beta+1}\right),
\end{aligned}
$$

where in the last step we used (4.14). Similarly,

$$
\sum_{1 \leqslant i \leqslant \lambda_{1}} \phi\left(\mu_{n, i}-i+\lambda_{1}\right)=\sum_{1 \leqslant i \leqslant \lambda_{1}} \phi\left(\mu_{n, i}\right)+\sum_{1 \leqslant i \leqslant \lambda_{1}} \bar{R}_{\phi, i}
$$

where the error term satisfies $\left|\bar{R}_{\phi, i}\right| \leqslant\left(\lambda_{1}-i\right) \ln \left(\mu_{n, i}-i+\lambda_{1}\right)$, and so by (4.14) and (4.10)

$$
\left|\sum_{1 \leqslant i \leqslant \lambda_{1}} \bar{R}_{\phi, i}\right|=o_{p}\left(\frac{1}{h_{n}^{(\beta+3) / 2}}\right)
$$


On the other hand, we have by (4.10) and (4.26)

$$
\sum_{i>\lambda_{1}} \phi\left(\mu_{n, i}\right)=\sum_{i>\lambda_{1}}\left(\mu_{n, i} \ln \mu_{n, i}-\mu_{n, i}\right)=O_{p}\left(\frac{1}{h_{n}}(\ln n)^{\beta+2}\right) .
$$

Combining the preceding, (4.22) becomes

$$
\begin{aligned}
\ln N_{n}= & \sum_{1 \leqslant i \leqslant \lambda_{1}}\left(\lambda_{i}^{\prime}-\mu_{n, i}\right) \ln \Psi_{\beta}\left(i h_{n}\right)+\frac{\beta+1}{\beta+2} \ln n ! \\
& -b_{n}+o_{p}\left(\frac{1}{h_{n}^{(\beta+3) / 2}}\right),
\end{aligned}
$$

where $b_{n}$ is as in (4.4).

It remains to prove that the summation term in the right-hand side of (4.26) satisfies the central limit theorem. In fact,

$$
\sum_{1 \leqslant i \leqslant \lambda_{1}}\left(\lambda_{i}^{\prime}-\mu_{n, i}\right) \ln \Psi_{\beta}\left(i h_{n}\right)=\int_{0}^{\infty}\left(\lambda_{[x]}^{\prime}-\mu_{n,[x]}\right) \ln \Psi_{\beta}\left(x h_{n}\right) d x+R_{n},
$$

where $R_{n}$ is bounded by

$$
\sum_{i=1}^{\infty}\left|\lambda_{i}^{\prime}-\mu_{n, i}\right|\left(\ln \Psi_{\beta}\left((i-1) h_{n}\right)-\ln \Psi_{\beta}\left(i h_{n}\right)\right)=o_{p}\left(\frac{1}{h_{n}^{(\beta+3) / 2}}\right) .
$$

Moreover, a change of variable gives

$$
h_{n}^{(\beta+3) / 2} \int_{0}^{\infty}\left(\lambda_{[x]}^{\prime}-\mu_{n,[x]}\right) \ln \Psi_{\beta}\left(x h_{n}\right) d x=\int_{0}^{\infty} V_{n}(x) \ln \Psi_{\beta}(x) d x .
$$

Therefore, we have by (4.21) and (4.26)

$$
h_{n}^{(\beta+3) / 2}\left(\ln \frac{d_{\lambda^{\prime}}}{(n !)^{1 /(\beta+2)}}-b_{n}\right)=-\int_{0}^{\infty} V_{n}(x) \ln \Psi_{\beta}(x) d x+o_{p}(1) .
$$

Now a direct application of Theorem 3.3 finishes the proof of (4.20). Theorem 4.1 is proved.

Acknowledgement. The author expresses his gratitude to the anonymous referee for his careful reading and insightful comments, which substantially improved the paper.

\section{REFERENCES}

1. Andrews G.E. The Theory of Partitions, Encyclopedia of Mtahematics and Its Applications, Vol. 2, Addison-Wesley, Reading, MA, 1976.

2. Биллингсли П. Сходимость вероятностных мер. М.: Наука, 1977, 352 с.

3. Bloch S., Okounkov A. The character of the infinite wedge representation. - Adv. Math., 2000, v. 149, № 1, p. 1-60. 
4. Bogachev L.V., Su Z. G. Gaussian fluctuations of Young diagrams under the Plancherel measure. - Proc. R. Soc. Lond. Ser. A Math. Phys. Eng. Sci., 2007, v. 463, p. 1069-1080.

5. Borodin A., Okounkov A., Olshanski G. Asymptotics of Plancherel measures for symmetric groups. - J. Amer. Math. Soc., 2000, v. 13, № 3, p. 481-515.

6. de Bruijn N. G. Asymptotic Methods in Analysis. Amsterdam: North-Holland Publishing, 1958.

7. Erdös P., Lehner J. The distribution of the number of summands in the partition of a positive integer. - Duke Math. J., 1941, v. 8, p. 335-345.

8. Erlihson M. M., Granovsky B.L. Limit shapes of Gibbs distributions on the set of integer partitions: The expansive case. - Ann. Inst. H. Poincare Probab. Statist., 2008, v. 44, № 5, p. 915-945.

9. Frame J.S., de B. Robinson G., Thrall R. M. The hook graphs of the symmetric groups. - Canadian J. Math., 1954, v. 6, p. 316-324.

10. Fristedt $B$. The structure of random partitions of large integers. - Trans. Amer. Math. Soc., 1993, v. 2, № 2, p. 703-735.

11. Frobenius $G$. Uber die charactere der symmetrischen gruppe. - Sitzungsber. Preuss. Akad. Berlin, 1903, p. 328-358.

12. Гихман И.И., Скороход А.В. Введение в теорию случайных процессов. М.: Наука, 1965, 656 с.

13. Ivanov V., Olshanski G. Kerov's central limit theorem for the Plancherel measure on Young diagrams. Dordrecht: Kluwer Academic Publishers, 2002.

14. Johansson $K$. Discrete orthogonal polynomial ensembles and the Plancherel measure. - Ann. Math., 2001, v. 153, № 1, p. 259-296.

15. Kerov S. Gaussian limit for the Plancherel measure of the symmetric group. - C. R. Acad. Sci. Paris Ser. I Math., 1993, v. 316, № 4, p. 303-308.

16. Logan B.F., Shepp L. A. A variational problem for random Young tableaux. - Adv. Math., 1977, v. 26, № 2, p. 206-222.

17. Okounkov A. Random matrices and random permutations. - Internat. Math. Res. Notices, 2000, v. 20, p. 1043-1095.

18. Okounkov A. The uses of random partitions. XIVth International Congress on Mathematical Physics, Jean-Claude Zambrini ed., World Sci. Publ., 2005, p. 379-403.

19. Петров В. В. Суммы независмых случайных величин. М.: Наука, 1972, 416 с.

20. Pittel B. On a likely shape of the random Ferrers digram. - Adv. Appl. Math., 1997, v. 18, № 4, p. 432-488.

21. Pittel B. On the distribution of the number of Young tableaux for a uniformly random diagram. - Adv. Appl. Math., 2002, v. 29, № 2, p. 184-214.

22. Szalay M., Turán P. On some problems of the statistical theory of partitions with applications to characters of the symmetric group. - Acta Math. Acad. Sci. Hungar. I, 1997, v. 29, p. 361-379; II, p. 381-392; III, 1978, v. 32, p. 129-155.

23. Vershik A.M. Statistical mechanics of combinatorial partitions, and their limit shapes. - Funct. Anal. Appl., 1996, v. 30, № 2, p. 90-105.

24. Vershik A.M. Limit shapes of typical geometric configurations and their applications. - J. Math. Sci., 2004, v. 119, № 2, p. 165-177.

25. Vershik A.M., Kerov S. Asymptotics of the Plancherel measure of the symmetric group and the limit form of Young tableaux. — Soviet Math. Dokl., 1977, v. 18, p. $527-531$.

26. Vershik A.M., Yakubovich Yu. Fluctuations of the maximal particle energy of the quantum ideal gas and random partitions. — Comm. Math. Phys., 2006, v. 261, № 3, p. $795-769$.

Поступила в редакцию 6.IV.2011

Исправленный вариант 6.VII.2011 